\title{
Time-Domain Goal-Oriented Adaptivity Using Pseudo-Dual Error Representations
}

\author{
Judit Muñoz-Matute ${ }^{\mathrm{a}}$, Elisabete Alberdi ${ }^{\mathrm{a}}$, \\ David Pardo ${ }^{\mathrm{a}, \mathrm{b}, \mathrm{c}}$, Victor M. Calo ${ }^{\mathrm{d}, \mathrm{e}}$ \\ ${ }^{a}$ University of the Basque Country (UPV/EHU), Leioa, Spain \\ ${ }^{b}$ BCAM-Basque Center for Applied Mathematics, Bilbao, Spain \\ ${ }^{c}$ IKERBASQUE, Basque Foundation for Science, Bilbao, Spain \\ ${ }^{d}$ Applied Geology, Western Australian School of Mines, Faculty of Science and \\ Engineering, Curtin University, Perth, WA, Australia 6845 \\ ${ }^{e}$ Mineral Resources, Commonwealth Scientific and Industrial Research Organisation \\ (CSIRO), Kensington, WA, Australia 6152
}

\begin{abstract}
Goal-oriented adaptive algorithms produce optimal grids to solve challenging engineering problems. Recently, a novel error representation using (unconventional) pseudo-dual problems for goal-oriented adaptivity in the context of frequency-domain wave-propagation problems has been developed. In this paper, we extend this error representation to the case of time-domain problems. We express the entire problem in weak form in order to derive the adjoint formulation and apply goal-oriented adaptivity. One dimensional (1D) numerical results show that upper bounds for the new error representation are sharper than the classical ones. Therefore, this new error representation can be used to design more efficient goal-oriented adaptive methodologies.
\end{abstract}

Keywords: wave equation, goal-oriented adaptivity, error representation, Finite Element Method.

\section{Introduction}

The phenomenon of wave propagation and its applications is present in our daily lives and its study is essential to improve our quality of life and to better understand nature. Examples of wave propagation problem include acoustics, elasticity (stress waves in solids), fluid and gas dynamics (shock wave propagation), and electromagnetics. 
Often, analytical solutions of the above problems are either unavailable or intractable, so it is essential to develop high-precision numerical methods to perform accurate simulations. Nowadays, the Finite Element Method (FEM) [1-4] is a commonly used technique to approximate solutions of partial differential equations. The flexibility of the geometric description that FEM affords, allows the methodology to model a wide variety of engineering problems.

Adaptive algorithms in FEM [5] are an essential tool to obtain accurate solutions without incurring an excessive computational cost. In these types of algorithms, the element size and/or the order of approximation vary locally. The adaptive processes use local indicators of the error that were initially designed to reduce the global error in the energy norm.

However, in many engineering problems, we need to accurately approximate a specific quantity of interest, i.e., some feature of the solution. From this need, the idea of "goal-oriented adaptivity" emerged. The seminal works developed by Becker and Rannacher $[6,7]$ and the subsequent contributions of Oden and Prudhomme [8-11] have been of great importance in this area, which has been applied now to a wide variety of problems [12-16]. In the goal-oriented approach, the quantity of interest is often expressed in terms of a functional of the solution, and the error in the quantity of interest is represented using the errors of the original and dual problems. Subsequently, an upper bound of the error representation is usually expressed in terms of local contributions of the bilinear form of the problem, which is further bounded by a sum of local error norms. The resulting upper bounds can be used as the main criteria to perform adaptivity [17], since they decrease under proper mesh refinements.

Recently, Darrigrand et. al. [18] developed a new error representation within the goal-oriented approach for frequency-domain problems. They obtained sharper upper bounds than the classical ones for the Helmholtz equation in 1D. Their method is based on employing an alternative bilinear form that exhibits better properties than the original one. They represent the error in the quantity of interest using both this alternative form and the original one. Using this new approach, the existing adaptive processes for wave propagation problems can be improved.

The FEM and the adaptive processes in time-dependent problems are different from frequency-domain methods. The approximations in evolution problems (such as the heat or the wave equations) are often calculated with the Method of Lines [19-21]. In this method, the space and time variables are discretized separately. First, the space variable is discretized using FEM and for that, a spatial-variational formulation [22-24] is needed. Once the 
space variable is discretized, a first or a second order system of Ordinary Differential Equations (ODEs) is obtained for the heat and the wave equation, respectively. Traditional numerical methods such as explicit Runge-Kutta methods or Backward Differentiation Formulae (BDF) [25-27] can be used for the resolution of first order ODEs. Second order ODE systems can be reduced to a first order ODE system and solved by the aforementioned numerical methods or directly using methods like the Hilber-Hughes-Taylor- $\alpha$ (HHT- $\alpha$ ) method $[28,29]$. The generalized- $\alpha$ methods are a robust family of time integration techniques with dissipative control on the highest resolved frequencies for first and second order discretizations [30, 31].

In the Method of Lines, authors perform the adaptivity independently in time and space $[32,33]$. However, for goal-oriented adaptivity, a variational formulation of the problem in both variables is essential in order to properly define the dual problem and the error in a quantity of interest. The concept of space-time variational formulation is explained in, for example, Bales and Lasiecka [34], French [35], Johnson [36], Hulbert and Hughes [37] for hyperbolic equations, and Morandi and Nociforo [38] for parabolic problems. Based on this formulation, goal-oriented adaptive strategies have been developed for parabolic and hyperbolic problems. In [39, 40] goal-oriented adaptivity is explained for structural transient dynamics; in [41-44] an error representation in the goal-oriented approach and corresponding upper bounds are derived for parabolic problems; and finally, in [45-47], Bangerth and Rannacher developed adaptive strategies for the wave equation.

In this work, we extend the new error representation developed in [18] to the $1 \mathrm{D}$ time-domain wave equation. By doing so, we generalize the existing goal-oriented adaptive algorithms for time-domain problems. We also obtain sharper upper bounds of the error representation in the goal-oriented approach for certain time-domain problems. This can be applied to better solve multiple wave propagation engineering problems, including acquisition of seismic images and interpretation of electromagnetic geophysical measurements $[48,49]$.

The remainder of this article is organized as follows. Section 2 describes the model problem used to illustrate the theory. We introduce a strong formulation and derive the corresponding variational formulation of the wave propagation problem in the time domain, and we also describe the discretization used. Section 3 introduces the classical error representation in a goal-oriented approach. In section 4 , we derive the new error representation applied to the wave equation. Section 5 describes two different adaptive algorithms, while section 6 describes the numerical results we obtain with the new error representation formula. Finally, section 7 summarizes our 
findings.

\section{Model Problem}

In this section, we describe the strong and weak formulations of the model problem that we study throughout this paper.

\subsection{Strong formulation}

Let $\Omega=(a, b) \subset \mathbb{R}$ and $I=(0, T] \subset \mathbb{R}$. We consider the following one-dimensional wave equation:

$$
\begin{cases}u_{t t}-\left(\alpha u_{x}\right)_{x}=f & \text { in } \Omega \times I, \\ u(a, t)=u(b, t)=0 & \text { in } I, \\ u(x, 0)=u_{0}(x) & \text { in } \Omega, \\ u_{t}(x, 0)=v_{0}(x) & \text { in } \Omega,\end{cases}
$$

where the speed of propagation of the wave $\sqrt{\alpha(x)}$, the source term $f(x, t)$, the initial position $u_{0}(x)$ and the initial speed $v_{0}(x)$ are given data that define the problem. We denote by $u_{x}$ and $u_{t}$ the partial derivatives of the solution $u(x, t)$ with respect to the spatial variable $x$ and the temporal variable $t$, respectively.

We assume that $f(x, t) \in L^{2}\left(I ; H^{-1}(\Omega)\right), u_{0}(x) \in H_{0}^{1}(\Omega)$ and $v_{0}(x) \in$ $L^{2}(\Omega)$, where $H_{0}^{1}(\Omega)=\left\{u \in H^{1}(\Omega) \mid u(a)=u(b)=0\right\}$ and $H^{-1}(\Omega)$ is its dual space. Here, $H^{1}(\Omega)=\left\{u \in L^{2}(\Omega) \mid \nabla u \in L^{2}(\Omega)\right\}$ with associated norm $\|u\|_{1}=\left(\int_{\Omega}\left(|\nabla u|^{2}+|u|^{2}\right)\right)^{1 / 2}$ and $L^{2}\left(I ; H_{0}^{1}(\Omega)\right)$ is a Bochner space, i.e., the space of all functions $u: I \longrightarrow H_{0}^{1}(\Omega)$ that are $H^{1}$-measurable and $\left(\int_{I}\|u(t)\|_{1}^{2} d t\right)^{1 / 2}<+\infty$

For simplicity, we will consider homogeneous Dirichlet conditions on the spatial boundaries.

\subsection{Variational formulation}

We perform the change of variables $v=u_{t}$, so equation (1) becomes

$$
\begin{cases}u_{t}=v & \text { in } \Omega \times I, \\ v_{t}-\left(\alpha u_{x}\right)_{x}=f & \text { in } \Omega \times I, \\ u(a, t)=u(b, t)=0 & \text { in } I, \\ u(x, 0)=u_{0}(x) & \text { in } \Omega, \\ v(x, 0)=v_{0}(x) & \text { in } \Omega .\end{cases}
$$


We know from [23] and [45] that the weak solution $\{u, v\}$ of problem (2) belongs to $\mathbb{U} \times \mathbb{V}$, where

$$
\begin{aligned}
& \mathbb{U}:=\left\{u \in L^{2}\left(I ; H_{0}^{1}(\Omega)\right) \mid u_{t} \in L^{2}\left(I ; L^{2}(\Omega)\right), u_{t t} \in L^{2}\left(I ; H^{-1}(\Omega)\right)\right\}, \\
& \mathbb{V}:=\left\{v \in L^{2}\left(I ; L^{2}(\Omega)\right) \mid v_{t} \in L^{2}\left(I ; H^{-1}(\Omega)\right)\right\},
\end{aligned}
$$

and it satisfies $u(x, 0)=u_{0}(x), v(x, 0)=v_{0}(x)$ and

$$
\begin{array}{r}
\left(u_{t}, \psi\right)-(v, \psi)=0, \forall \psi \in L^{2}(\Omega), \\
\left\langle v_{t}, \varphi\right\rangle+\left(\alpha u_{x}, \varphi_{x}\right)=\langle f, \varphi\rangle, \forall \varphi \in H_{0}^{1}(\Omega) .
\end{array}
$$

Here, $\langle\cdot, \cdot\rangle$ denotes the duality pairing between $H_{0}^{1}(\Omega)$ and $H^{-1}(\Omega)$, and $(\cdot, \cdot)$ denotes the inner product in $L^{2}(\Omega)$.

Using the spaces defined above, the weak solution $\{u, v\}$ is continuous in time

$$
u \in \mathcal{C}\left(\bar{I} ; H_{0}^{1}(\Omega)\right), \quad v \in \mathcal{C}\left(\bar{I} ; L^{2}(\Omega)\right) .
$$

Now, we select the following test spaces

$$
\mathbb{W}:=L^{2}\left(I ; L^{2}(\Omega)\right), \quad \mathbb{Z}:=L^{2}\left(I ; H_{0}^{1}(\Omega)\right),
$$

and integrating in time the expressions in (3) and imposing the initial conditions in weak form, we conclude that (3) is equivalent to

$$
\begin{array}{r}
\int_{I}\left(u_{t}, \psi\right) d t-\int_{I}(v, \psi) d t+(u(0), \psi(0))=\left(u_{0}, \psi(0)\right), \forall \psi \in \mathbb{W}, \\
\int_{I}\left\langle v_{t}, \varphi\right\rangle d t+\int_{I}\left(\alpha u_{x}, \varphi_{x}\right) d t+(v(0), \varphi(0))= \\
=\int_{I}\langle f, \varphi\rangle d t+\left(v_{0}, \varphi(0)\right), \forall \varphi \in \mathbb{Z},
\end{array}
$$

where $u(0):=u(x, 0)$ and $v(0):=v(x, 0)$.

We write the above expressions in a compact form by adding the two equations in (4), denoting by $U:=\{u, v\}, \Psi:=\{\psi, \varphi\}$, and defining:

$$
\begin{aligned}
& B(U, \Psi):=\int_{I}\left(u_{t}, \psi\right) d t-\int_{I}(v, \psi) d t+(u(0), \psi(0)) \\
&+\int_{I}\left\langle v_{t}, \varphi\right\rangle d t+\int_{I}\left(\alpha u_{x}, \varphi_{x}\right) d t+(v(0), \varphi(0)), \\
& F(\Psi):=\left(u_{0}, \psi(0)\right)+\int_{I}\langle f, \varphi\rangle d t+\left(v_{0}, \varphi(0)\right),
\end{aligned}
$$


and finally, the variational formulation of problem (2) becomes

$$
\| \begin{gathered}
\text { Find } U=\{u, v\} \in \mathbb{U} \times \mathbb{V} \text { such that } \\
\qquad(U, \Psi)=F(\Psi), \\
\text { for all } \Psi=\{\psi, \varphi\} \in \mathbb{W} \times \mathbb{Z},
\end{gathered}
$$

where $F(\cdot)$ is linear and $B(\cdot, \cdot)$ is bilinear.

\subsection{Discretization}

In order to approximate the solution of (5), we use a finite element discretization in space and time. First, we define a mesh over the spatial domain, i.e., we perform a partition of the interval $\Omega=(a, b)$ into $n$ subintervals as follows

$$
a=x_{0}<x_{1}<\cdots<x_{n-1}<x_{n}=b,
$$

and we denote by $\Omega_{i}=\left(x_{i-1}, x_{i}\right), h_{i}=x_{i}-x_{i-1}, \forall i=1, \ldots, n$ and $h=$ $\max _{1 \leq i \leq n} h_{i}$.

Related to this partition, we denote by $V_{h}$ the finite-dimensional subspace of $H_{0}^{1}(\Omega)$ generated by the continuous, piecewise linear functions defined over each subinterval.

Similarly, for the time interval $I=(0, T]$, we select

$$
0=t_{0}<t_{1}<\ldots<t_{m-1}<t_{m}=T \text {. }
$$

We denote $k_{j}=t_{j}-t_{j-1}, \forall j=1, \ldots, m$ and $k=\max _{1 \leq j \leq m} k_{j}$.

In order to discretize problem (5), we select the following discrete spaces

$$
\begin{aligned}
\mathcal{U}_{h k} & :=\left\{u \in C\left(\bar{I} ; V_{h}\right) \mid u_{\left.\right|_{I_{j}}} \in P_{1}\left(I_{j} ; V_{h}\right), \forall j=1, \ldots, m\right\}, \\
\mathcal{W}_{h k} & :=\left\{u \in L^{2}\left(I ; V_{h}\right) \mid u_{I_{I_{j}}} \in P_{0}\left(I_{j} ; V_{h}\right), \forall j=1, \ldots, m\right\},
\end{aligned}
$$

where $I_{j}=\left(t_{j-1}, t_{j}\right], \forall j=1, \ldots, m, C\left(\bar{I} ; V_{h}\right)$ is the Banach space of continuous functions that takes values in $V_{h}$ and $P_{r}\left(I_{j} ; V_{h}\right)$ is the space of all polynomials with degree less than or equal to $r$ on the interval $I_{j}$ with values in $V_{h}$. The functions in $\mathcal{U}_{h k}$ are piecewise linear in space and time and globally continuous, whereas functions in $\mathcal{W}_{h k}$ are continuous piecewise linear in space and discontinuous piecewise constant in time. 
Finally, the discrete variational formulation becomes

$$
\begin{aligned}
& \text { Find } U_{h k}=\left\{u_{h k}, v_{h k}\right\} \in \mathcal{U}_{h k} \times \mathcal{U}_{h k} \text { such that } \\
& \qquad B\left(U_{h k}, \Psi_{h k}\right)=F\left(\Psi_{h k}\right), \\
& \text { for all } \Psi_{h k}=\left\{\psi_{h k}, \varphi_{h k}\right\} \in \mathcal{W}_{h k} \times \mathcal{W}_{h k} .
\end{aligned}
$$

\section{Goal-oriented adaptivity}

There are two key ingredients in goal-oriented adaptivity $[8,10,11]$ :

1) an error representation formula that enables to subsequently bound the error in the quantity of interest as a sum of normed errors over the elements, and

2) a posteriori error estimation to approximate the values of the aforementioned bounds.

In this paper, we focus only on 1), while we assume that 2) is given, as it occurs for example in the adaptive processes followed in [17, 48, 49].

\subsection{Output functionals and the dual problem}

We first define the concepts of output functional and dual problem. The quantity of interest is given by a functional on the solution space

$$
L: \mathbb{U} \times \mathbb{V} \longrightarrow \mathbb{R} .
$$

For simplicity, we will only consider linear output functionals of the form

$$
L(U)=L_{0}(u)+L_{1}(v),
$$

where $L_{0}: \mathbb{U} \longrightarrow \mathbb{R}$ and $L_{1}: \mathbb{V} \longrightarrow \mathbb{R} . L(\cdot)$ is called the output functional and it represents a physical quantity of the solution.

We now introduce an auxiliary dual problem used to represent the error in the quantity of interest:

$$
\begin{aligned}
& \text { Find } \Psi=\{\psi, \varphi\} \in \mathbb{W} \times \mathbb{Z} \text { such that } \\
& B(U, \Psi)=L(U), \\
& \text { for all } U=\{u, v\} \in \mathbb{U} \times \mathbb{V} \text {. }
\end{aligned}
$$


The dual problem provides information about how the error in every spacetime point influences the error in the quantity of interest.

If the form of the functional is

$$
L_{0}(u)=\int_{I}\langle u, g\rangle d t+\left(u(T), \psi_{T}\right), L_{1}(v)=\left(v(T), \varphi_{T}\right),
$$

where $g \in L^{2}\left(I ; H^{-1}(\Omega)\right), \psi_{T} \in L^{2}(\Omega)$ and $\varphi_{T} \in H_{0}^{1}(\Omega)$ are given functions, we see that separating the dual problem in terms of test functions $u$ and $v$, and integrating by parts in time, we obtain

$$
\begin{gathered}
(u(T), \psi(T))-\int_{I}\left\langle u, \psi_{t}\right\rangle d t+\int_{I}\left(u_{x}, \alpha \varphi_{x}\right) d t=\int_{I}\langle u, g\rangle d t+\left(u(T), \psi_{T}\right), \\
(v(T), \varphi(T))-\int_{I}\left(v, \varphi_{t}\right) d t-\int_{I}(v, \psi) d t=\left(v(T), \varphi_{T}\right),
\end{gathered}
$$

and the corresponding strong formulation of dual problem (7) is

$$
\begin{cases}\varphi_{t}=-\psi & \text { in } \Omega \times I, \\ -\psi_{t}-\left(\alpha \varphi_{x}\right)_{x}=g & \text { in } \Omega \times I, \\ \varphi(a, t)=\varphi(b, t)=0 & \text { in } I, \\ \varphi(x, T)=\varphi_{T}(x) & \text { in } \Omega, \\ \psi(x, T)=\psi_{T}(x) & \text { in } \Omega .\end{cases}
$$

We conclude from (8) that the dual problem is also a wave propagation problem, but running backwards in time.

\subsection{Error representation}

In order to provide an error representation for the traditional goaloriented approach, we consider the continuous and discrete primal problems

Find $U^{*}=\left\{u^{*}, v^{*}\right\} \in \mathbb{U} \times \mathbb{V}$ and $U_{h k}^{*}=\left\{u_{h k}^{*}, v_{h k}^{*}\right\} \in \mathcal{U}_{h k} \times \mathcal{U}_{h k}$ such that

$$
\begin{gathered}
B\left(U^{*}, \Psi\right)=F(\Psi), \quad \forall \Psi=\{\psi, \varphi\} \in \mathbb{W} \times \mathbb{Z}, \\
B\left(U_{h k}^{*}, \Psi_{h k}\right)=F\left(\Psi_{h k}\right), \quad \forall \Psi_{h k}=\left\{\psi_{h k}, \varphi_{h k}\right\} \in \mathcal{W}_{h k} \times \mathcal{W}_{h k},
\end{gathered}
$$

and their duals

Find $\Psi^{*}=\left\{\psi^{*}, \varphi^{*}\right\} \in \mathbb{W} \times \mathbb{Z}$ and $\Psi_{h k}^{*}=\left\{\psi_{h k}^{*}, \varphi_{h k}^{*}\right\} \in \mathcal{W}_{h k} \times \mathcal{W}_{h k}$ such that

$$
\begin{gathered}
B\left(U, \Psi^{*}\right)=L(U), \quad \forall U=\{u, v\} \in \mathbb{U} \times \mathbb{V}, \\
B\left(U_{h k}, \Psi_{h k}^{*}\right)=L\left(U_{h k}\right), \quad \forall U_{h k}=\left\{u_{h k}, v_{h k}\right\} \in \mathcal{U}_{h k} \times \mathcal{U}_{h k} .
\end{gathered}
$$


Here, we use symbol * to denote the solution of the problem in order to avoid confusion between solution and test functions.

We define the errors of the primal and dual problems as follows

$e_{h k}:=U^{*}-U_{h k}^{*}=\left\{u^{*}-u_{h k}^{*}, v^{*}-v_{h k}^{*}\right\}, \quad \varepsilon_{h k}:=\Psi^{*}-\Psi_{h k}^{*}=\left\{\psi^{*}-\psi_{h k}^{*}, \varphi^{*}-\varphi_{h k}^{*}\right\}$.

Since $\mathcal{W}_{h k} \times \mathcal{W}_{h k}$ is a subspace of $\mathbb{W} \times \mathbb{Z}$, equation (9) also holds for all functions in $\mathcal{W}_{h k} \times \mathcal{W}_{h k}$, so we can substitute $\Psi_{h k}$ in (9). Now, substracting from it equation (10), and using the bilinearity of $B(\cdot, \cdot)$ we obtain an expression of the Galerkin orthogonality

$$
B\left(e_{h k}, \Psi_{h k}\right)=0, \quad \forall \Psi_{h k} \in \mathcal{W}_{h k} \times \mathcal{W}_{h k}
$$

Following the same proccess with (11) and $U_{h k}$, we obtain

$$
B\left(U_{h k}, \varepsilon_{h k}\right)=0, \quad \forall U_{h k} \in \mathcal{U}_{h k} \times \mathcal{U}_{h k} .
$$

Now, replacing $U$ by $e_{h k}$ in (11) and using the Galerkin orthogonality (13), we obtain the classical error representation

$$
L\left(e_{h k}\right)=B\left(e_{h k}, \varepsilon_{h k}\right) .
$$

The above expression represents the error in the quantity of interest in terms of an integral over the whole domain.

If we denote by $K:=\Omega_{i} \times I_{j}$ to each space-time element and $B_{K}(\cdot, \cdot)$ to the restriction of the bilinear form $B(\cdot, \cdot)$ to each element $K$, we obtain the following upper bound of the error in terms of local element contributions

$$
\left|L\left(e_{h k}\right)\right|=\left|B\left(e_{h k}, \varepsilon_{h k}\right)\right|=\left|\sum_{K} B_{K}\left(e_{h k}, \varepsilon_{h k}\right)\right| \leq \sum_{K}\left|B_{K}\left(e_{h k}, \varepsilon_{h k}\right)\right| .
$$

Equation (16) can still be further bounded by a sum over $\left\|e_{h k}\right\|\left\|_{K}\right\| \varepsilon_{h k} \|_{K}$, where $\|\cdot\|_{K}$ is a suitable norm stemming from operator $B_{K}$ (in the case where $B_{K}$ is positive definite, one would directly select its associated norm). From the mathematical point of view, this sum of norms is the one that should be minimized, but from the engineering point of view, minimizing $\sum_{K}\left|B_{K}\left(e_{h k}, \varepsilon_{h k}\right)\right|$ often provides better adaptive algorithms (fewer unknowns are needed to achieve a given tolerance error), since this upper bound is sharper than the standard norm-based upper bound [48]. In this work, we display results corresponding to this engineering approach, although we obtain similar results when we consider the upper bound given in terms of $\sum_{K}\left\|e_{h k}\right\|_{K}\left\|\varepsilon_{h k}\right\|_{K}$ 


\section{Goal-oriented adaptivity using pseudo-dual problems}

In this section, we apply the pseudo-dual error representation of Darrigrand et al. [18] to the wave equation.

\subsection{New error representation}

By linearity, we have that $B\left(U, \varepsilon_{h k}\right)=B\left(U, \Psi^{*}\right)-B\left(U, \Psi_{h k}^{*}\right)$, and from (11) we obtain a weak formulation for the error of the dual problem

$$
\begin{aligned}
& \text { Find } \varepsilon_{h k} \in \mathbb{W} \times \mathbb{Z} \text { such that } \\
& B\left(U, \varepsilon_{h k}\right)=L(U)-B\left(U, \Psi_{h k}^{*}\right), \\
& \text { for all } U \in \mathbb{U} \times \mathbb{V} \text {. }
\end{aligned}
$$

Following [18], we select an alternative bilinear form $\tilde{B}(\cdot, \cdot)$ in $(17)$ in order to obtain a new error $\tilde{\varepsilon}_{h k}$ as the solution of the following problem

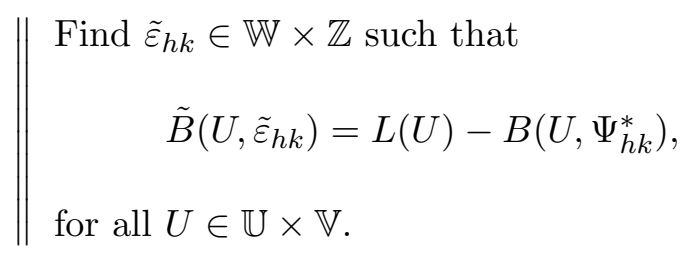

From (12), we see that this alternative bilinear form also satisfies the Galerkin orthogonality

$$
\tilde{B}\left(U_{h k}, \tilde{\varepsilon}_{h k}\right)=L\left(U_{h k}\right)-B\left(U_{h k}, \Psi_{h k}^{*}\right)=0, \quad \forall U_{h k} \in \mathcal{U}_{h k} \times \mathcal{U}_{h k} .
$$

Now, replacing $U$ by $e_{h k}$ in (18) and using the Galerkin orthogonality (13), we obtain the new error representation

$$
L\left(e_{h k}\right)=\tilde{B}\left(e_{h k}, \tilde{\varepsilon}_{h k}\right),
$$

and, as in (16), we have the upper bound

$$
\left|L\left(e_{h k}\right)\right|=\left|\tilde{B}\left(e_{h k}, \tilde{\varepsilon}_{h k}\right)\right|=\left|\sum_{K} \tilde{B}_{K}\left(e_{h k}, \tilde{\varepsilon}_{h k}\right)\right| \leq \sum_{K}\left|\tilde{B}_{K}\left(e_{h k}, \tilde{\varepsilon}_{h k}\right)\right| .
$$

In [18], the authors show numerically that for the Helmholtz equation in $1 \mathrm{D}$, there exist alternative bilinear forms for which the upper bounds of the new error representation are sharper than the classical ones. This fact leads us to think that if we find a suitable alternative bilinear form $\tilde{B}(\cdot, \cdot)$, the 
upper bound (20) could also be sharper than (16) for time-domain problems. The resulting method is a better guidance criterion for the adaptive process.

Following [18], we select the alternative bilinear form $\tilde{B}(\cdot, \cdot)$ by modifying from $B(\cdot, \cdot)$ the terms coming from the second order time derivative

$$
\begin{array}{r}
\tilde{B}(U, \Psi)=\beta \int_{I}\left(u_{t}, \psi\right) d t-\int_{I}(v, \psi) d t+\beta(u(0), \psi(0)) \\
+\beta \int_{I}\left\langle v_{t}, \varphi\right\rangle d t+\int_{I}\left(\alpha u_{x}, \varphi_{x}\right) d t+\beta(v(0), \varphi(0)),
\end{array}
$$

where $\beta \in \mathbb{R}-\{0\}$.

Following an analogous process to that described in Section 3, we conclude that the strong formulation of the unconventional dual problem

$$
\begin{aligned}
& \| \text { Find } \Psi=\{\psi, \varphi\} \in \mathbb{W} \times \mathbb{Z} \text { such that } \\
& \tilde{B}(U, \Psi)=L(U), \\
& \text { for all } U=\{u, v\} \in \mathbb{U} \times \mathbb{V} \text {. }
\end{aligned}
$$

is given by

$$
\begin{cases}\beta \varphi_{t}=-\psi & \text { in } \Omega \times I, \\ -\beta \psi_{t}-\left(\alpha \varphi_{x}\right)_{x}=g & \text { in } \Omega \times I, \\ \varphi(a, t)=\varphi(b, t)=0 & \text { in } I, \\ \beta \varphi(x, T)=\varphi_{T}(x) & \text { in } \Omega, \\ \beta \psi(x, T)=\psi_{T}(x) & \text { in } \Omega .\end{cases}
$$

We know that the solution of the homogeneous wave equation $u_{t t}-$ $\alpha u_{x x}=0$ is of the form

$$
u(x, t)=\sum_{n=0}^{\infty}\left(c_{n} \cos \left(\frac{n \pi \sqrt{\alpha} t}{l}\right)+d_{n} \sin \left(\frac{n \pi \sqrt{\alpha} t}{l}\right)\right) \sqrt{\frac{2}{l}} \sin \left(\frac{n \pi x}{l}\right)
$$

where $l$ is the length of the spatial interval $\Omega=(a, b)$ and $c_{n}$ and $d_{n}$ are constants that depend on the initial conditions of the equation.

Problem (23) verifies equation $\varphi_{t t}-\frac{1}{\beta^{2}}\left(\alpha \varphi_{x}\right)_{x}=\frac{1}{\beta^{2}} g$, so when $\beta$ is large enough, the solution of (23) converges to the solution of its associated homogeneous equation. Moreover, when $\beta$ is large enough, $\varphi_{t t}$ is close to zero, and therefore, the solution $\varphi(x, t)$ is linear in time. In conclusion, with the alternative bilinear form (21), we are selecting unconventional dual problems that are almost linear in time. 


\section{Refinement strategies}

In this section, we describe the two refinement algorithms used in this work in order to compare the upper bounds given by (16) and (20). Although we know the analytical solution of the wave equation (1), we do not know the solutions of problems (11) and (18). Therefore, we work with two meshes: a coarse mesh and a reference mesh. We perform optimal refinements over the coarse mesh, while the reference mesh is employed to calculate the reference solutions to problems (9) and (11).

\subsection{Uniform hk-refinements}

In Algorithm 1, we perform uniform refinements in space (h-refinements) and time (k-refinements) simultaneously. The inputs of the algorithm are the endpoints $a$ and $b$ of $\Omega$, the final instant $T$, the number of global refinements $n_{\text {refin }}$ and the number of elements in space $n_{\text {coarse }}$ and time $m_{\text {coarse }}$ of the coarse mesh. First, we calculate the primal (9) and dual (11) reference solutions. We solve the primal problem (10) and dual problem (12) in the coarse mesh and also in a sequence of uniformly refined meshes. Then, we inject the primal and dual solutions over the reference mesh. According to the discrete spaces selected in section 2, the injection of the primal problem is performed by piecewise linear interpolation both in space and time whereas the injection of the dual problem is performed by piecewise linear interpolation in space and piecewise constant interpolation in time. Finally, we estimate the errors $e_{h k}$ and $\varepsilon_{h k}$ and solve the unconventional dual problem (18) to estimate $\tilde{\varepsilon}_{h k}$. 


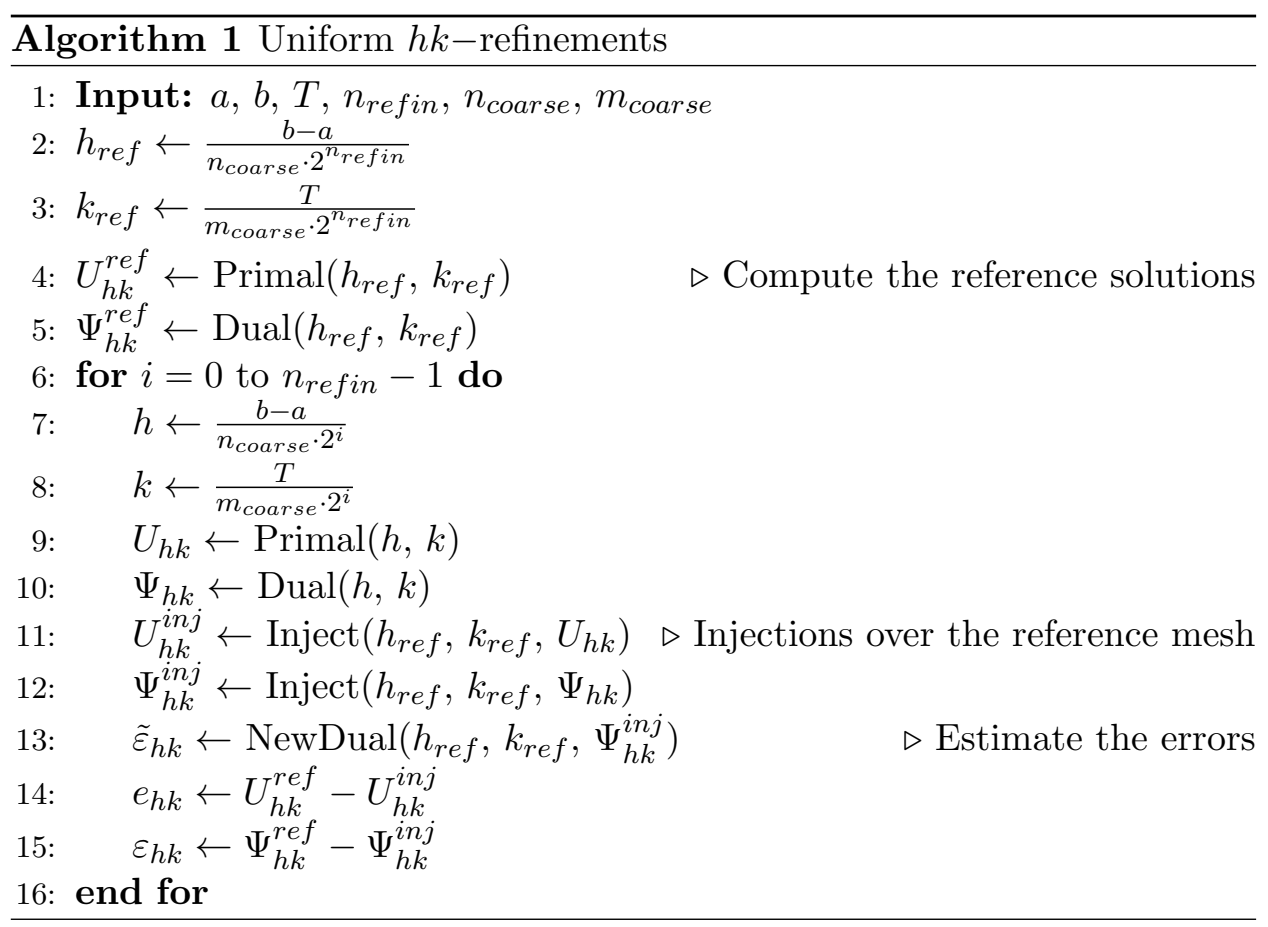

\subsection{Goal-oriented $h$ - and k-adaptivity}

Algorithm 2 performs local refinements in space for all $t \in I$ and, simultaneously, local refinements in time for all $x \in \Omega$. In other words, we restrict to tensor-product type refinements. We use the following upper bound of the error in terms of local contributions in time and space separately

$$
\left|L\left(e_{h k}\right)\right|=\left|B\left(e_{h k}, \varepsilon_{h k}\right)\right| \leq \frac{1}{2} \sum_{i=1}^{n}\left|B_{\Omega_{i}}\left(e_{h k}, \varepsilon_{h k}\right)\right|+\frac{1}{2} \sum_{j=1}^{m}\left|B_{I_{j}}\left(e_{h k}, \varepsilon_{h k}\right)\right|,
$$

where $B_{\Omega_{i}}(\cdot, \cdot)$ and $B_{I_{j}}(\cdot, \cdot)$ are restrictions of the bilinear form $B(\cdot, \cdot)$ to each element $\Omega_{i} \times I$ and $\Omega \times I_{j}$, respectively.

The inputs of the algorithm are the endpoints $a$ and $b$, the final instant $T$, the tolerances $t o l_{1}$ and $t o l_{2}$, and the number of elements in space $n, n_{\text {ref }}$ and time $m, m_{\text {ref }}$ of the coarse and reference meshes, respectively.

First, we calculate the primal (9) and dual (11) reference solutions. Then, we solve the primal (10) and dual (12) problems in the coarse mesh and we estimate the relative error in the quantity of interest, which is used as a stopping criterion. For each element, we estimate the following quantities 
according to the upper bound (24)

$$
\begin{gathered}
\frac{\left|B_{\Omega_{i}}\left(e_{h k}, \varepsilon_{h k}\right)\right|}{\max \left(\max _{\Omega_{i}}\left|B_{\Omega_{i}}\left(e_{h k}, \varepsilon_{h k}\right)\right|, \max _{I_{j}}\left|B_{I_{j}}\left(e_{h k}, \varepsilon_{h k}\right)\right|\right)} \cdot 100, \\
\frac{\left|B_{I_{j}}\left(e_{h k}, \varepsilon_{h k}\right)\right|}{\max \left(\max _{\Omega_{i}}\left|B_{\Omega_{i}}\left(e_{h k}, \varepsilon_{h k}\right)\right|, \max _{I_{j}}\left|B_{I_{j}}\left(e_{h k}, \varepsilon_{h k}\right)\right|\right)} \cdot 100 .
\end{gathered}
$$

We refine those elements in which the above quantities are greater than or equal to tol $_{2}$. Finally, the obtained adapted mesh becomes the coarse grid for the next iteration. The adaptive process ends when the relative error of $L(U)$ is below $t_{0} l_{1}$ or when $h$ or $k$ of the adapted mesh is smaller than $h_{r e f}$ or $k_{r e f}$ of the reference mesh, that is, when the refinement process reaches the resolution of the reference solution in either space or time.

Using (24), we consider a new upper bound

$$
\left|L\left(e_{h k}\right)\right|=\left|\tilde{B}\left(e_{h k}, \tilde{\varepsilon}_{h k}\right)\right| \leq \frac{1}{2} \sum_{i=1}^{n}\left|\tilde{B}_{\Omega_{i}}\left(e_{h k}, \tilde{\varepsilon}_{h k}\right)\right|+\frac{1}{2} \sum_{j=1}^{m}\left|\tilde{B}_{I_{j}}\left(e_{h k}, \tilde{\varepsilon}_{h k}\right)\right| .
$$

Then, we employ the following quantities as the criteria to guide the adaptive process in Algorithm 2

$$
\begin{gathered}
\frac{\left|\tilde{B}_{\Omega_{i}}\left(e_{h k}, \tilde{\varepsilon}_{h k}\right)\right|}{\max \left(\max _{\Omega_{i}}\left|\tilde{B}_{\Omega_{i}}\left(e_{h k}, \tilde{\varepsilon}_{h k}\right)\right|, \max _{I_{j}}\left|\tilde{B}_{I_{j}}\left(e_{h k}, \tilde{\varepsilon}_{h k}\right)\right|\right)} \cdot 100, \\
\frac{\left|\tilde{B}_{I_{j}}\left(e_{h k}, \tilde{\varepsilon}_{h k}\right)\right|}{\max \left(\max _{\Omega_{i}}\left|\tilde{B}_{\Omega_{i}}\left(e_{h k}, \tilde{\varepsilon}_{h k}\right)\right|, \max _{I_{j}}\left|\tilde{B}_{I_{j}}\left(e_{h k}, \tilde{\varepsilon}_{h k}\right)\right|\right)} \cdot 100 .
\end{gathered}
$$




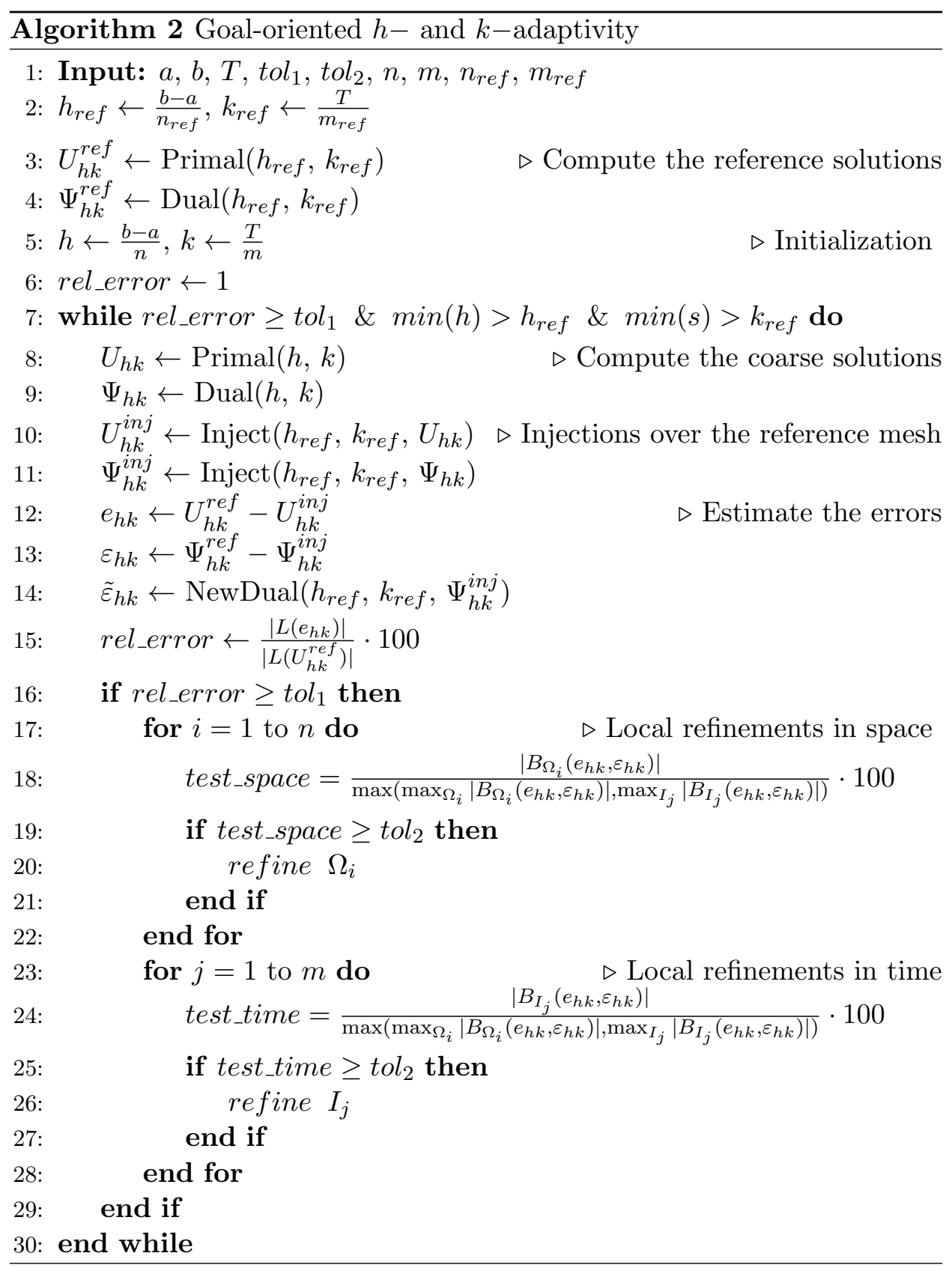




\section{Numerical results}

\subsection{Algorithm 1}

We consider the following problem: Let $\Omega=(-1,1)$ and $I=(0,2]$. We define

$$
\begin{cases}u_{t t}-\alpha u_{x x}=\alpha \cos (3 \pi t) & \text { in } \Omega \times I, \\ u(-1, t)=u(1, t)=0 & \text { in } I, \\ u(x, 0)=\phi(x) & \text { in } \Omega, \\ u_{t}(x, 0)=0 & \text { in } \Omega .\end{cases}
$$

where $\sqrt{\alpha}=\frac{3 \pi}{5}$ and

$$
\phi(x)=\frac{\cos (5 x) \sec (5)-1}{25} .
$$

We select the following output functional

$$
L_{0}(u)=\int_{I} \int_{\Omega} u(x, t) \alpha \cos (3 \pi t) d x d t, \quad L_{1}(v)=\int_{\Omega} v(x, T) \phi(x) d x .
$$

With this data, the source term of the dual problem is the same as in the primal problem and $\varphi(x, T)=u(x, 0)=\phi(x)$ (see Section 3), and therefore, the solutions of the primal and dual problems are such that $U(x, t)=\Psi(x, T-t)$, i.e, are the same function but reversed in time.

We set a coarse mesh with $2^{3}$ elements in space and time, respectively. Then, we perform 4 global uniform refinements so the reference mesh has $2^{7}$ elements in each variable $\left(2^{14}\right.$ elements in total). Figure 1 presents the primal (forward in time) solution, which coincides with the dual one that moves backwards in time.

Figure 2 shows the upper bounds obtained with a mesh of $2^{6}$ elements (the coarse one) when we vary the positive values of the parameter $\beta \in$ $\mathbb{R}-\{0\}$ of $\tilde{B}(\cdot, \cdot)$ described in Section 4 . We can see that when $\beta=1$ the bounds coincide, and when $\beta \in(0,1)$ the classical upper bound is, in general, sharper than the new one. This happens because the propagation speed of the wave and the source term of problem (23) are much higher so the numerical problem becomes unstable. However, when $\beta>1$, the pseudo-dual problem exhibits better stability properties than the classical dual problem as its speed of propagation is smaller and, for large values of $\beta$, the stability constant approaches one at the discrete level. We can see that when $\beta>1$, the new upper bound is sharper than the classical one, i.e.,

$$
\left|L\left(e_{h k}\right)\right| \leq \sum_{K}\left|\tilde{B}_{K}\left(e_{h k}, \tilde{\varepsilon}_{h k}\right)\right| \leq \sum_{K}\left|B_{K}\left(e_{h k}, \varepsilon_{h k}\right)\right| .
$$


Figure 3 shows a similar pattern for negative values of parameter $\beta$.

Figure 4 shows the solution of unconventional dual problem (22) and its derivative with $\beta=10^{2}$. We observe that the solution is constant in time and its derivative is close to zero.

Figures 5 to 7 show the errors of the primal, dual, and unconventional dual problems. Although the primal and dual solutions are the same, errors $e_{h k}$ and $\varepsilon_{h k}$ are different because they belong to different spaces. When $\beta=1$, errors $\varepsilon_{h k}$ and $\tilde{\varepsilon}_{h k}$ coincide because $\tilde{B}(\cdot, \cdot)=B(\cdot, \cdot)$ (see Section 3 ).

Finally, Figure 8 compares the upper bounds (16) and (20) of $\left|L\left(e_{h k}\right)\right|$ and we observe that for problem (32), the new upper bound is sharper than the classical one.

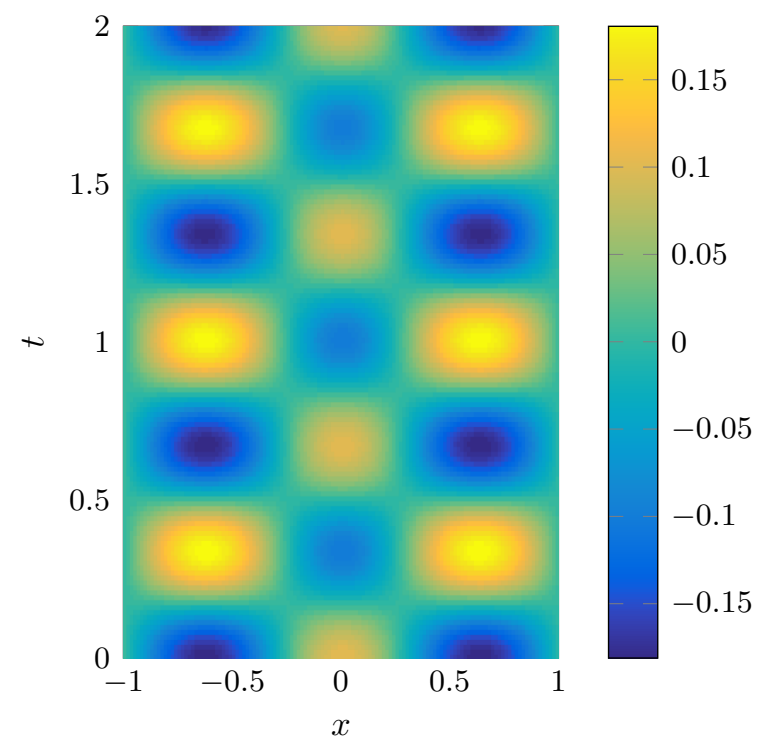

Figure 1: Colormap of the reference solution of the primal problem (forward in time). (The colormap coincides with the dual problem solution, which runs backwards in time). 


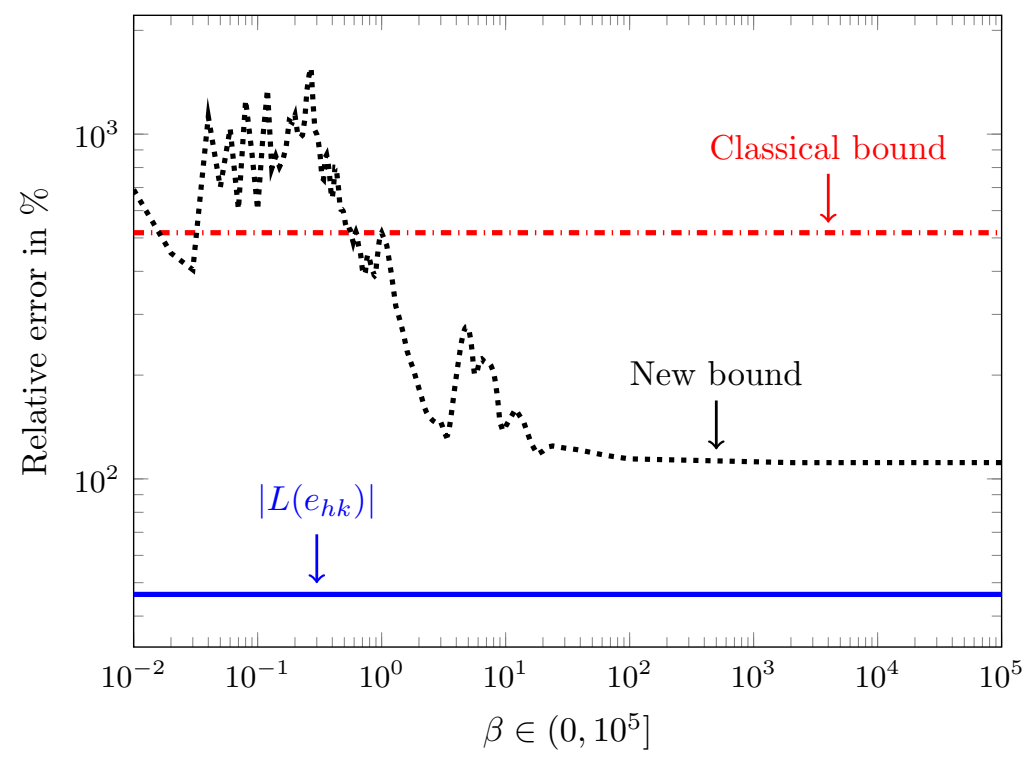

Figure 2: Upper bounds obtained in a given mesh and varying $\beta$ in the alternative bilinear form $\tilde{B}(\cdot, \cdot)$ in logarithmic scale.

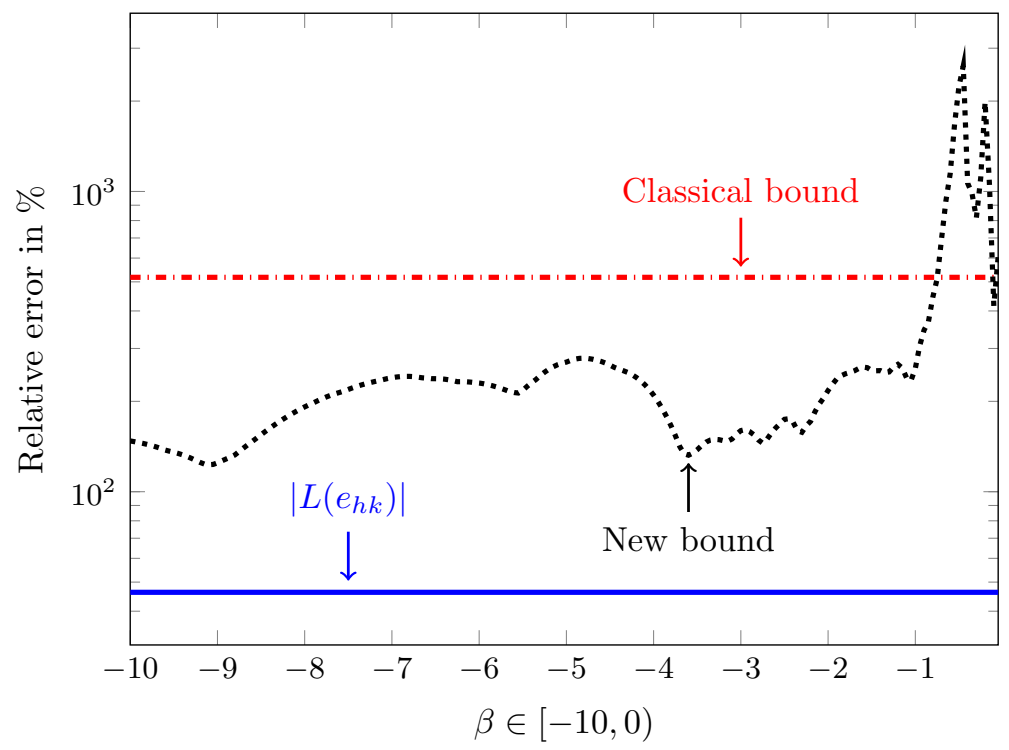

Figure 3: Upper bounds obtained in a given mesh and varying $\beta$ in the alternative bilinear form $\tilde{B}(\cdot, \cdot)$. 

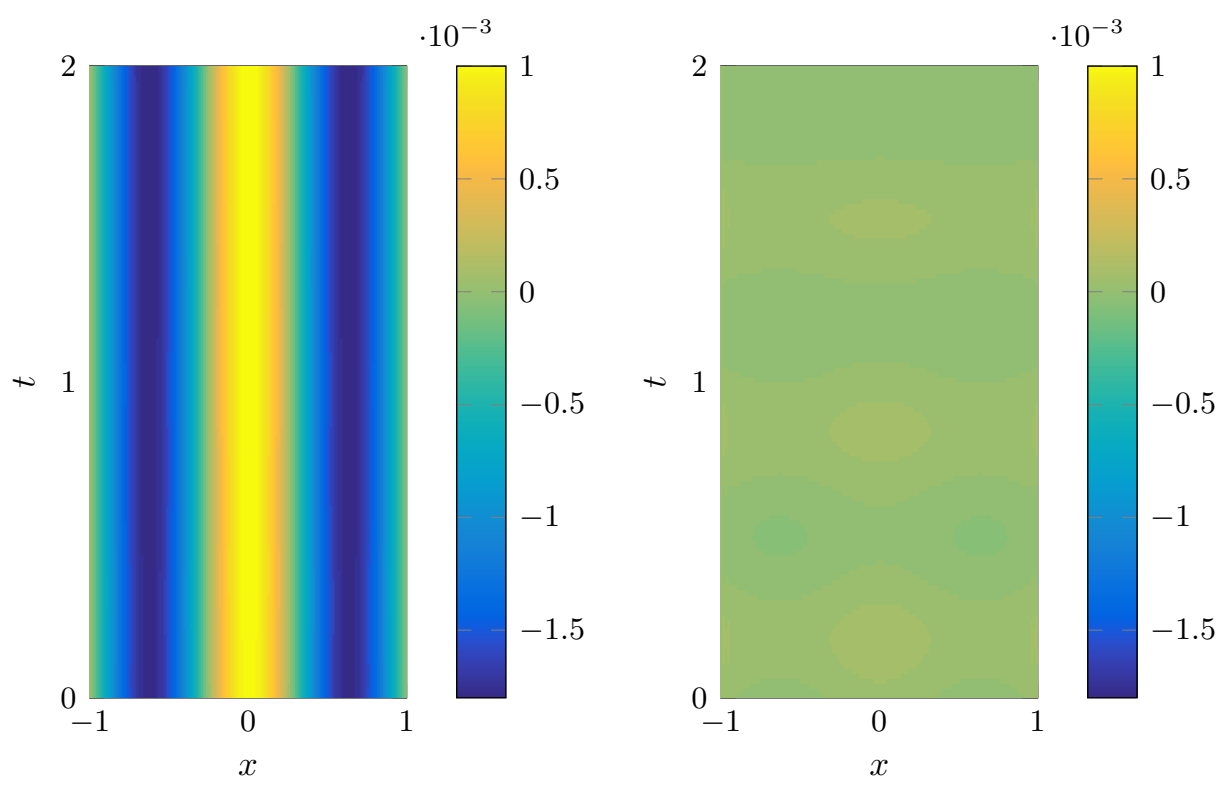

Figure 4: Colormap of the unconventional dual problem (22) (left) and its time derivative (right) with $\beta=10^{2}$. 


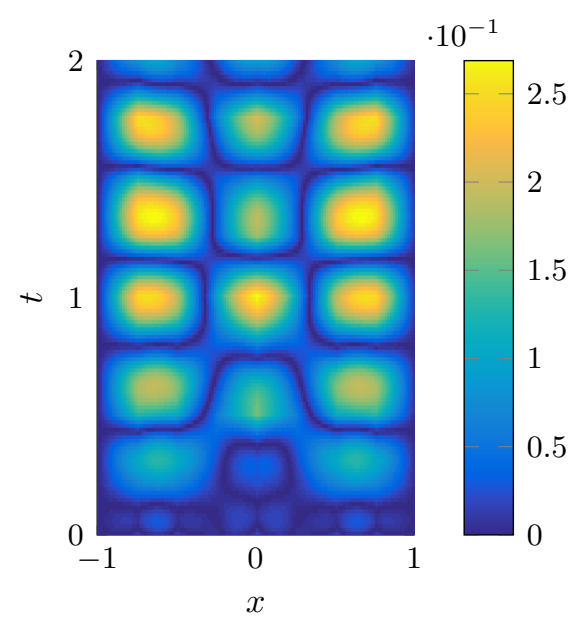

(a) Coarse mesh $\left|e_{h k}\right|$ error.

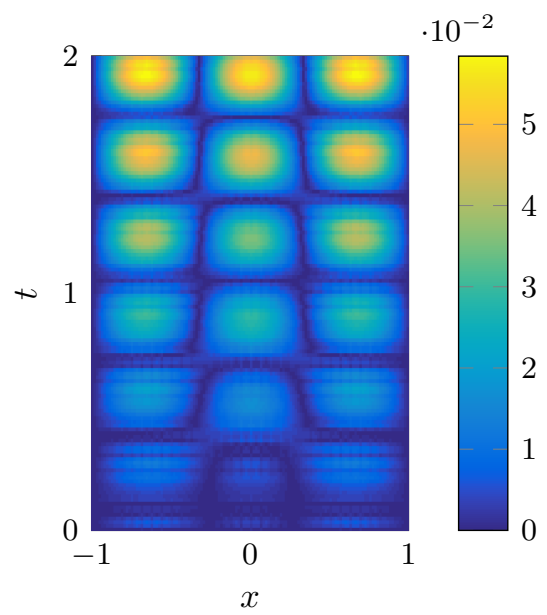

(c) Error $\left|e_{h k}\right|$ on mesh 2.

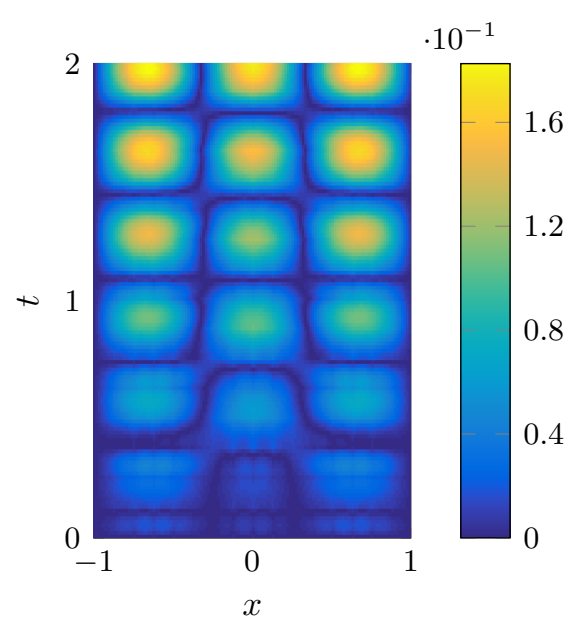

(b) Error $\left|e_{h k}\right|$ on mesh 1.

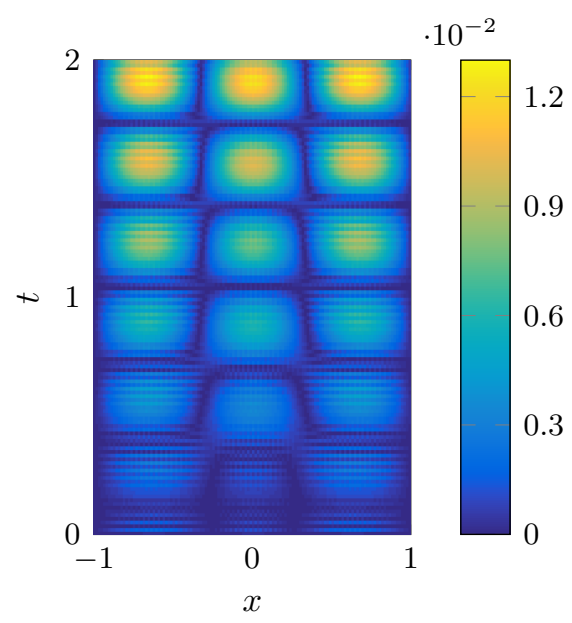

(d) Error $\left|e_{h k}\right|$ on mesh 3.

Figure 5: Colormap of the error of the primal problem with $\beta=10^{2}$ in different grids. 


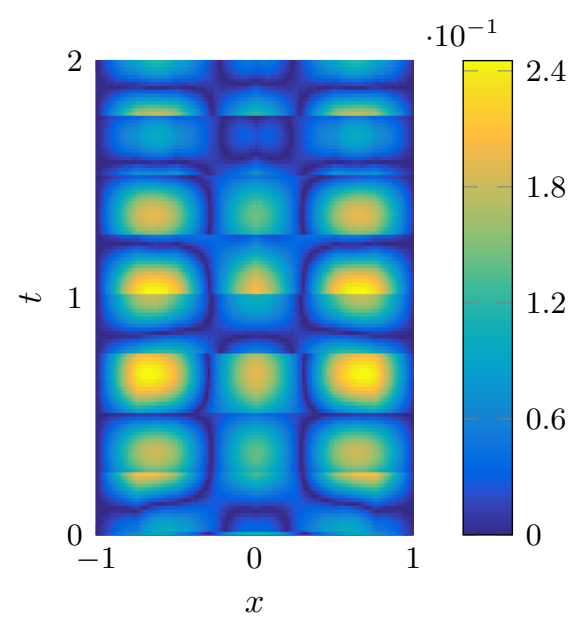

(a) Coarse mesh $\left|\varepsilon_{h k}\right|$ error.

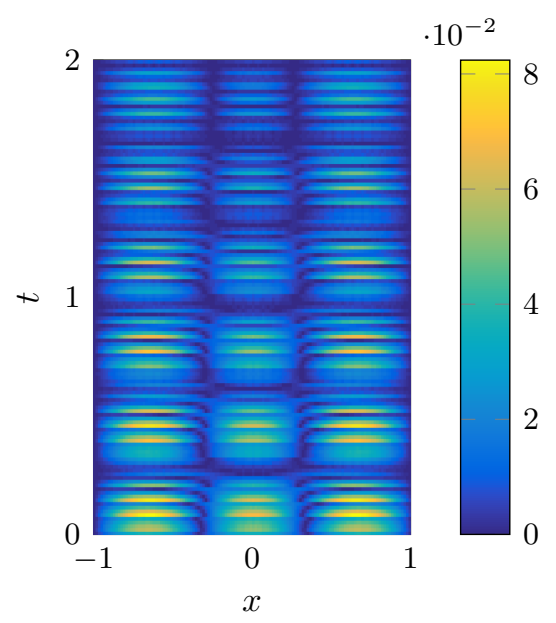

(c) Error $\left|\varepsilon_{h k}\right|$ on mesh 2 .

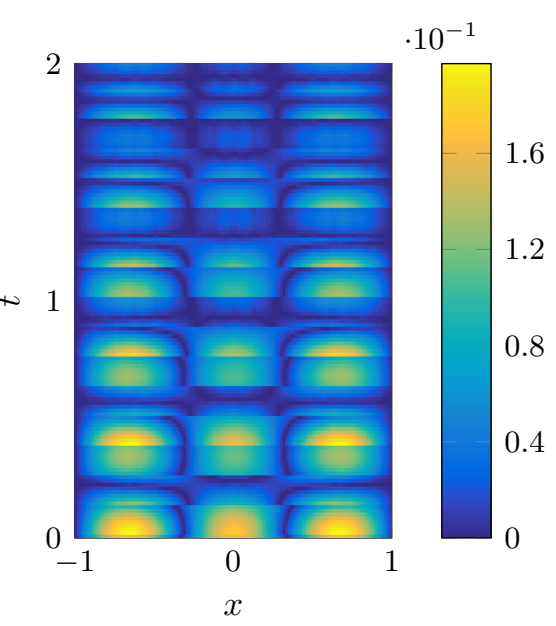

(b) Error $\left|\varepsilon_{h k}\right|$ on mesh 1.

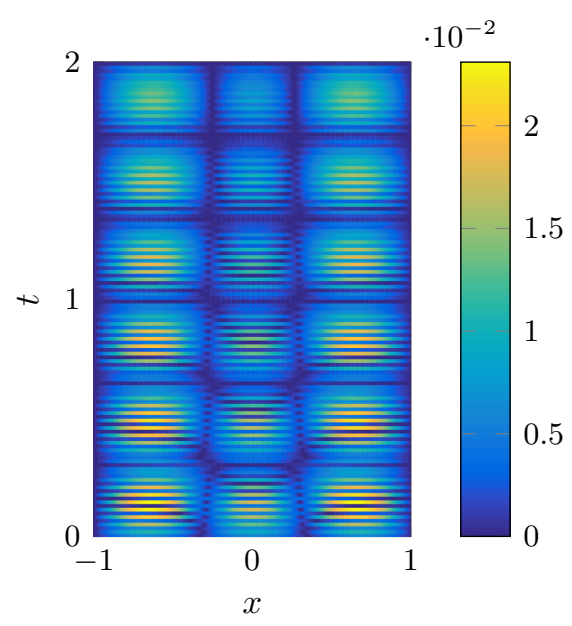

(d) Error $\left|\varepsilon_{h k}\right|$ on mesh 3.

Figure 6: Colormap of the error of the dual problem with $\beta=10^{2}$ in different grids. 


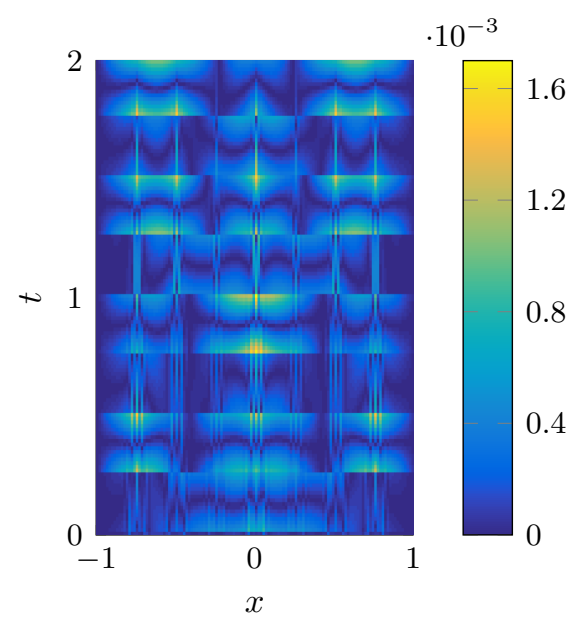

(a) Coarse mesh $\left|\tilde{\varepsilon}_{h k}\right|$ error.

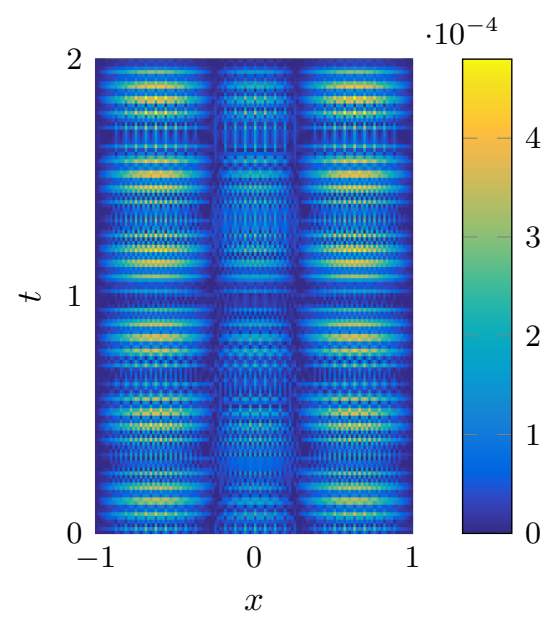

(c) Error $\left|\tilde{\varepsilon}_{h k}\right|$ on mesh 2.

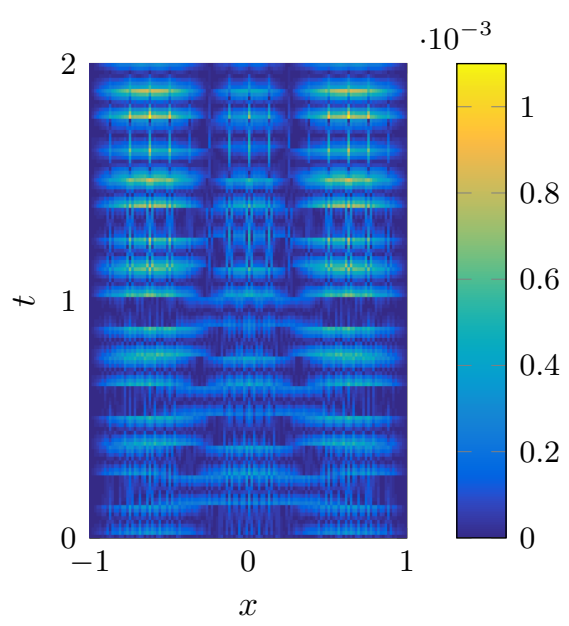

(b) Error $\left|\tilde{\varepsilon}_{h k}\right|$ on mesh 1 .

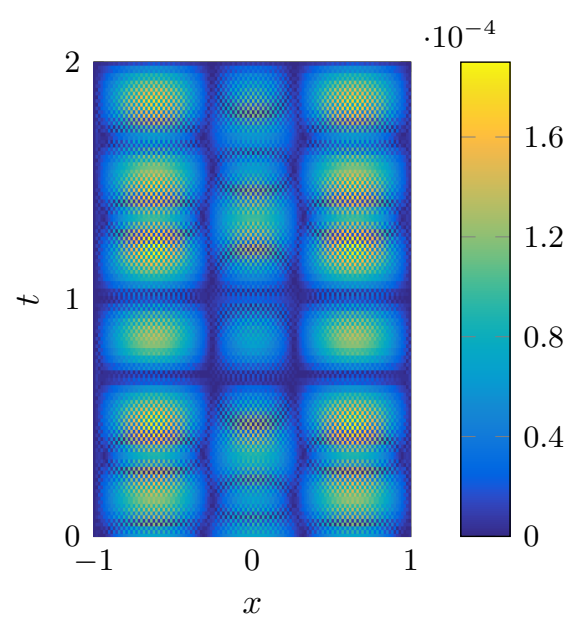

(d) Error $\left|\tilde{\varepsilon}_{h k}\right|$ on mesh 3.

Figure 7: Colormap of the solution of problem (18) with $\beta=10^{2}$ in different grids. 


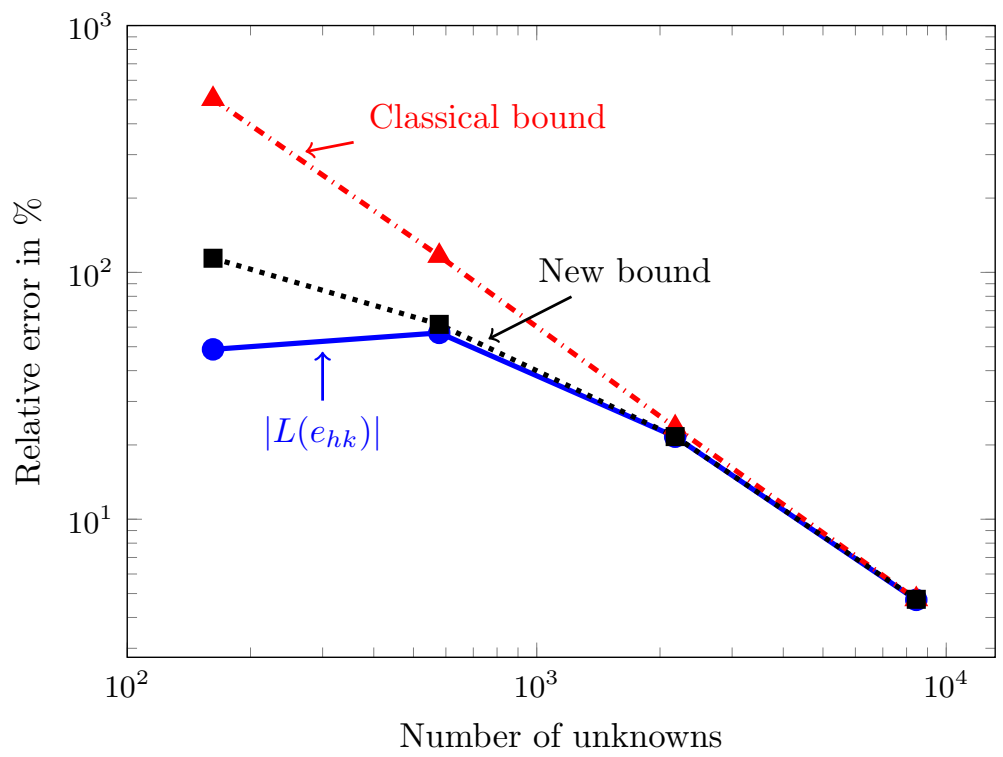

Figure 8: Upper bounds (16) and (20) with $\beta=10^{2}$.

Now, we consider the following output functional

$$
L_{0}(u)=\int_{I} \int_{\Omega_{0}} u(x, t) \cos (3 \pi t) d x d t
$$

where $\Omega_{0}=[-0.5,-0.25]$. In this case, the quantity of interest is a weighted solution over a small subdomain of $\Omega$.

Figure 9 displays the primal and dual reference solutions. Figures 10 and 11 show the errors of the dual and unconventional dual problems, respectively. Finally, Figure 12 exhibits the upper bounds. We conclude that for this case, the new upper bound is also sharper than the classical one. 

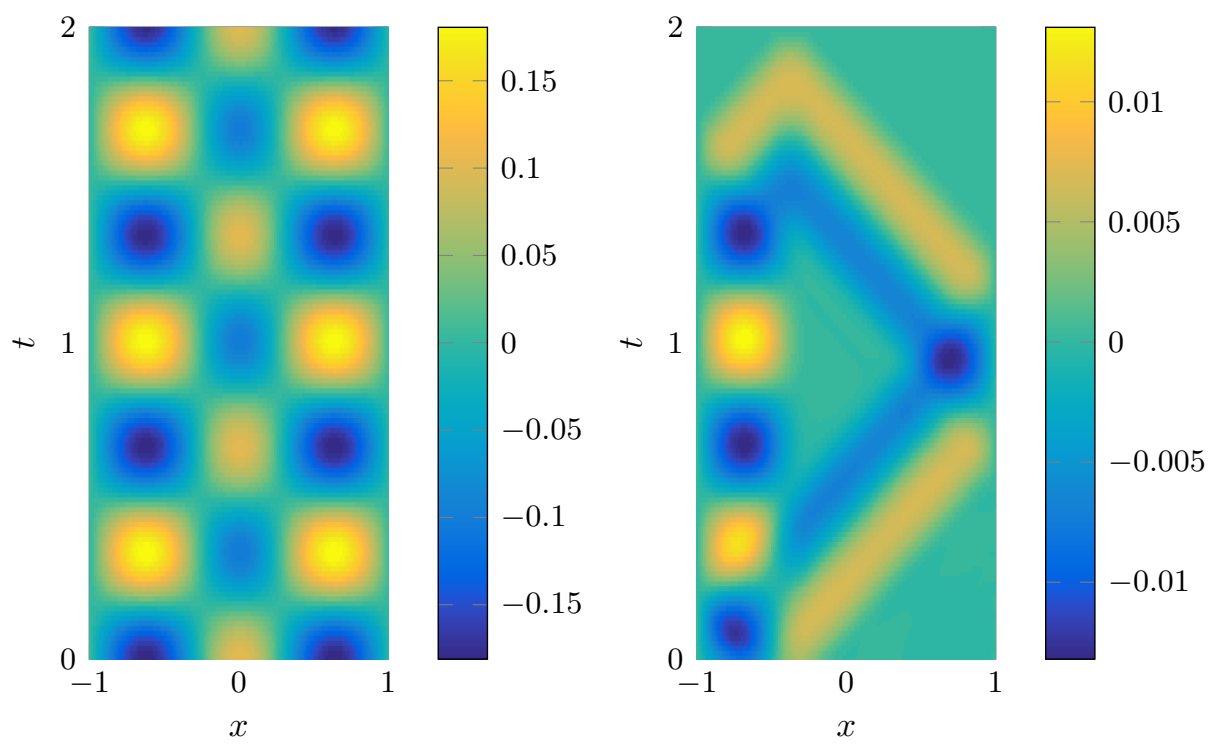

Figure 9: Colormap of the reference solution of the primal (left) and dual (right) problems. 


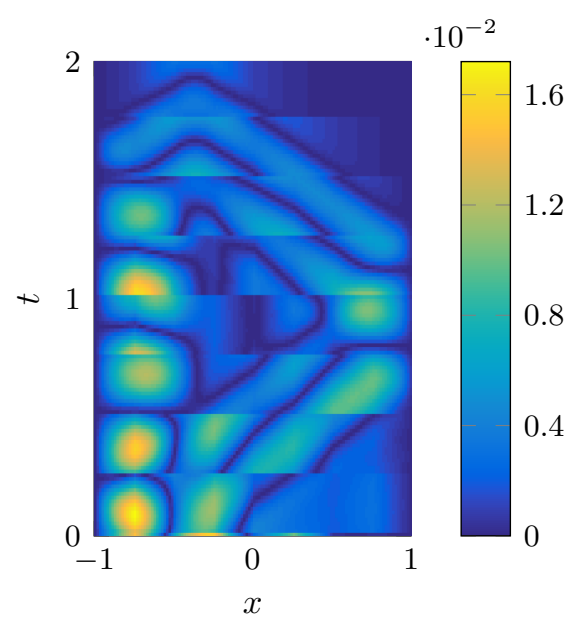

(a) Coarse mesh $\left|\varepsilon_{h k}\right|$ error.

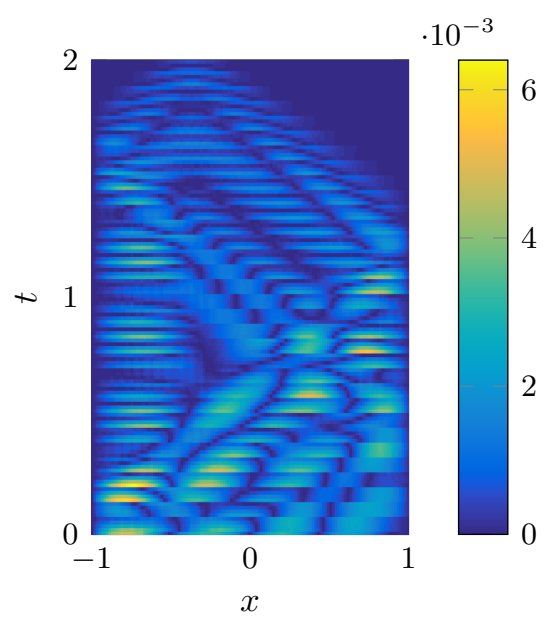

(c) Error $\left|\varepsilon_{h k}\right|$ on mesh 2.

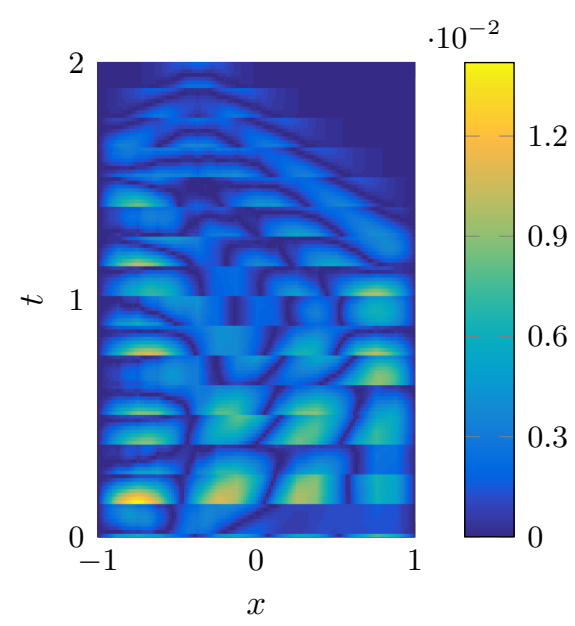

(b) Error $\left|\varepsilon_{h k}\right|$ on mesh 1.

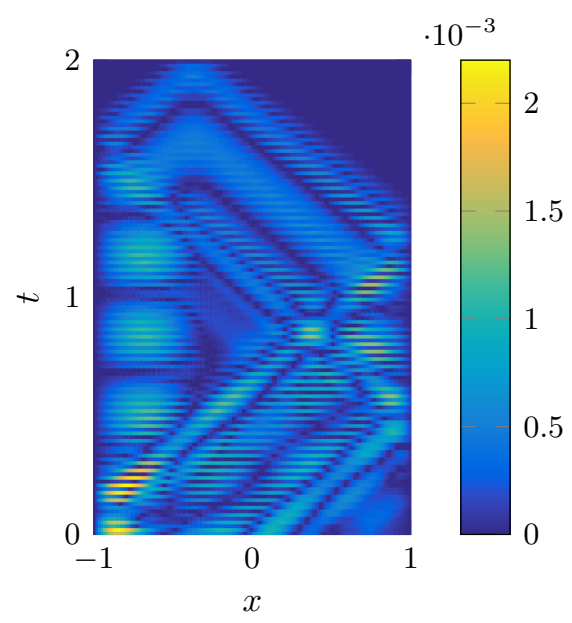

(d) Error $\left|\varepsilon_{h k}\right|$ on mesh 3.

Figure 10: Colormap of the error of the dual problem with $\beta=10^{2}$ in each refinement. 


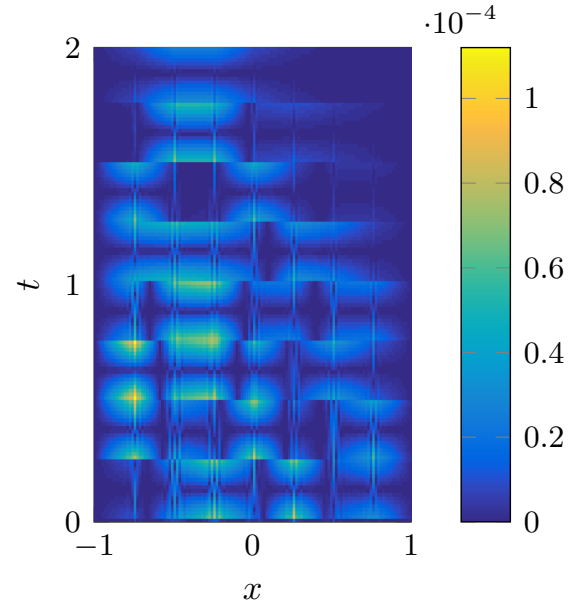

(a) Coarse mesh $\left|\tilde{\varepsilon}_{h k}\right|$ error.

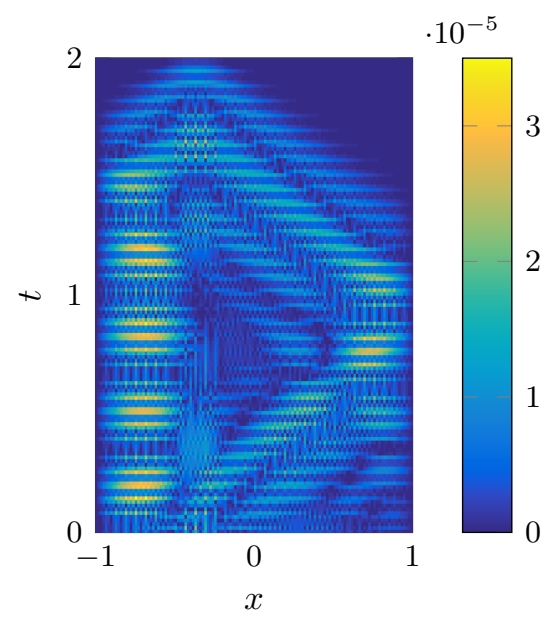

(c) Error $\left|\tilde{\varepsilon}_{h k}\right|$ on mesh 2.

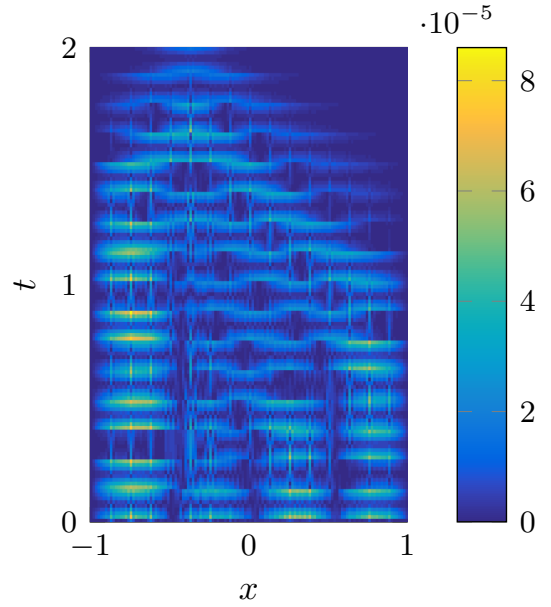

(b) Error $\left|\tilde{\varepsilon}_{h k}\right|$ on mesh 1.

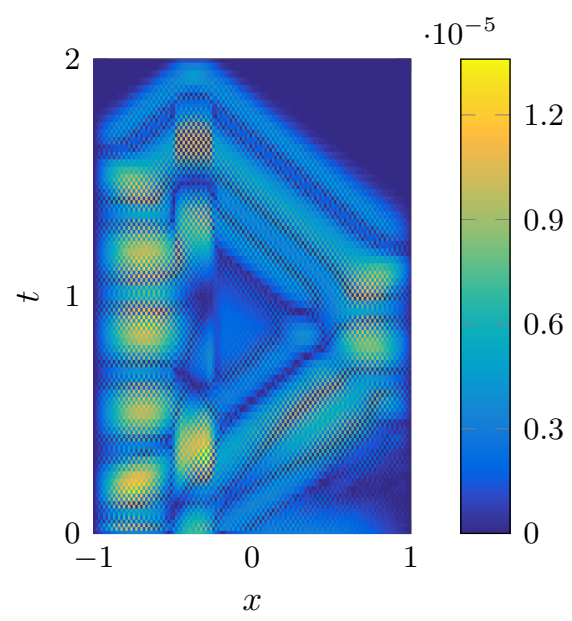

(d) Error $\left|\tilde{\varepsilon}_{h k}\right|$ on mesh 3.

Figure 11: Colormap of the solution of problem (18) with $\beta=10^{2}$ in each refinement. 


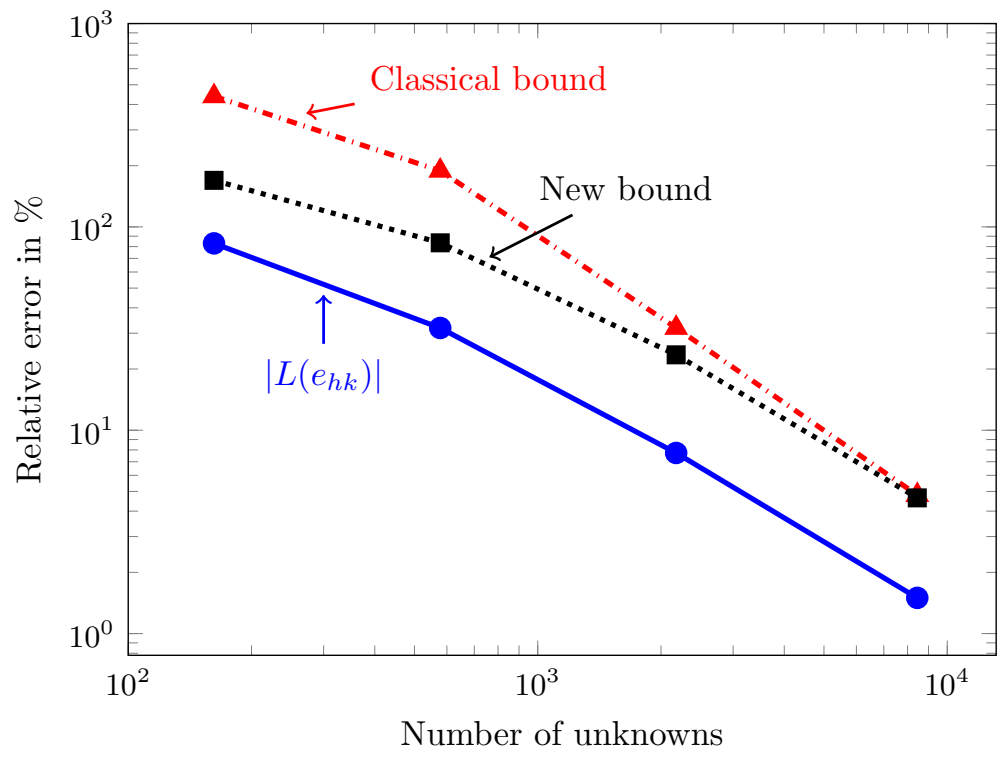

Figure 12: Upper bounds (16) and (20) with $\beta=10^{2}$. 


\subsection{Algorithm 2}

In this subsection, we consider the following problem: Let $\Omega=(-2,2)$ and $I=(0,6]$. We have

$$
\begin{cases}u_{t t}-\left(\alpha u_{x}\right)_{x}=f & \text { in } \Omega \times I, \\ u(-2, t)=u(2, t)=0 & \text { in } I, \\ u(x, 0)=0 & \text { in } \Omega, \\ u_{t}(x, 0)=0 & \text { in } \Omega,\end{cases}
$$

where the source term and the wave speed are defined piecewise as

$$
\begin{gathered}
f(x, t)= \begin{cases}2 \cos (2 \pi t), & x \in[0.5,1.5], \\
0 & t \in[0,1.5], \\
\text { elsewhere }\end{cases} \\
\alpha(x)= \begin{cases}\frac{1}{16}, & x \leq 0, \\
1 & x>0 .\end{cases}
\end{gathered}
$$

We consider the output functional

$$
L(u)=\int_{I} \int_{\Omega_{0}} \cos (2 \pi t) u(x, t) d x d t
$$

where $\Omega_{0}=[0.5,1.5]$. We select $\beta=10^{2}$ and perform the mesh adaptivity by executing Algorithm 2. We set a coarse mesh with $2^{6}$ elements and a reference mesh with $2^{14}$ elements. We also set the tolerances: $t_{0} l_{1}=0.5 \%$ and $t_{0} l_{2}=5 \%$.

Figure 14 shows the final adapted meshes when we use classical and alternative criterions to perform adaptivity. Figure 13 displays the primal and dual reference solutions. Figures 15 and 17 show the upper bounds (24) and (27) when we use the classical criteria (25) and (26), and the alternative criteria (28) and (29), respectively. In both cases, the new upper bound is sharper than the classical one. Finally, Figures 16 and 18 display the spatial and temporal contributions of the upper bounds (24) and (27). We conclude that, in this case, the temporal contribution is almost independent of the use of bound (24) or (27), but the spatial contribution is sharper when employing the new bound. 

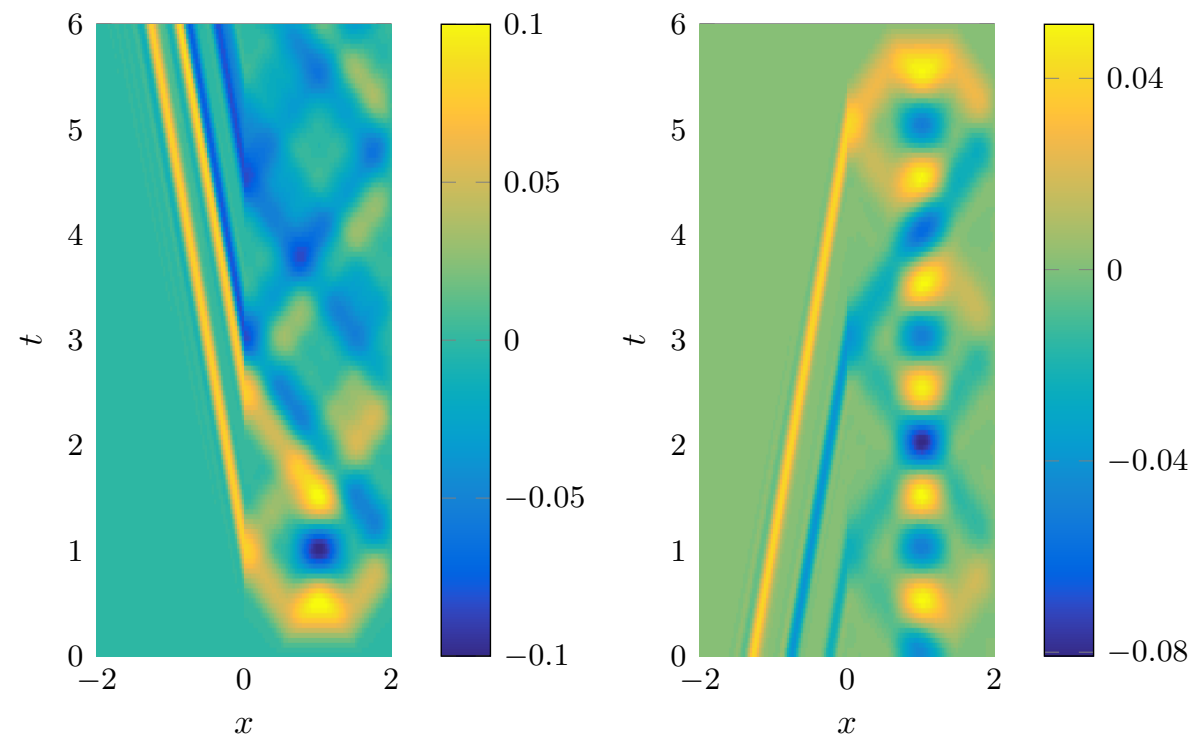

Figure 13: Colormap of the reference solution of the primal (left) and dual (right) problems.
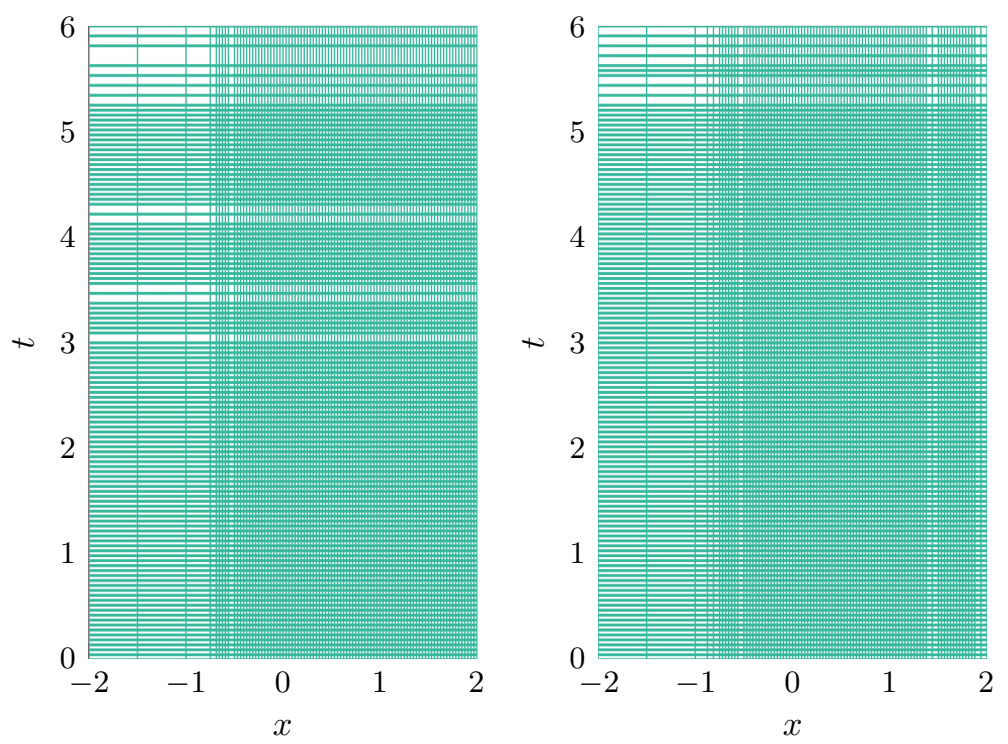

Figure 14: Resulting adapted meshes using classical criterion $((25),(26))$ (left) and alternative criterion $((28),(29))$ (right). 


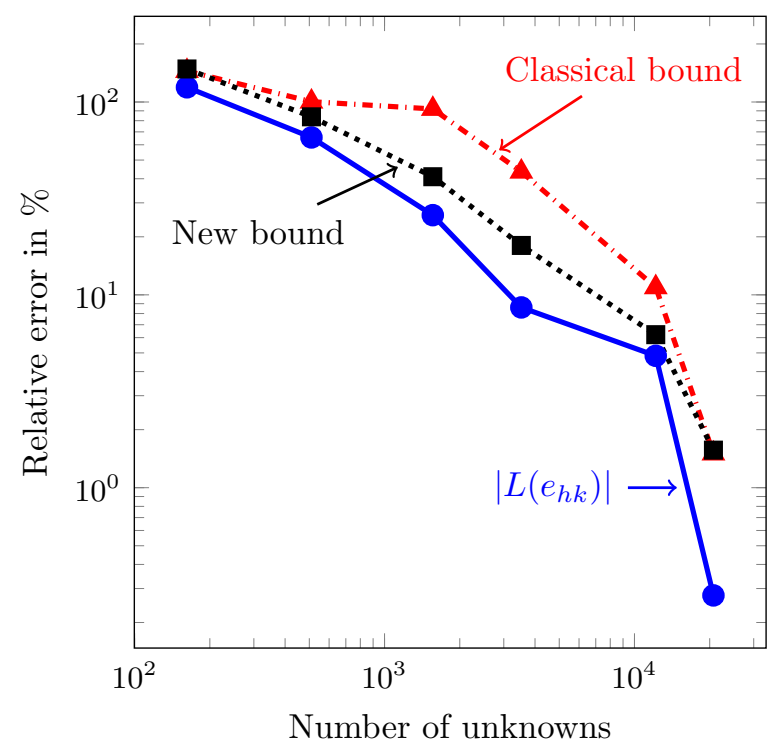

Figure 15: Upper bounds (24) and (27) corresponding to a goal-oriented adaptive algorithm using classical criterions (25) and (26).
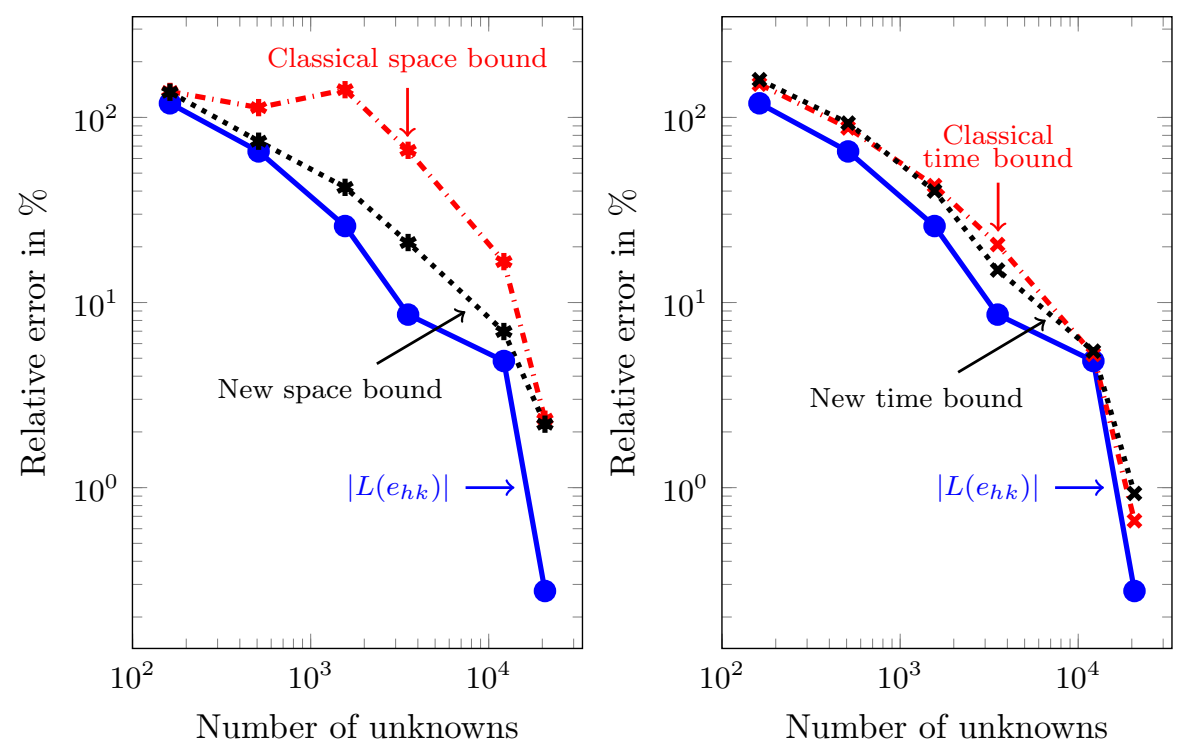

Figure 16: Spatial contribution (left) and temporal contribution (right) of the upper bounds (24) and (27) corresponding to a goal-oriented adaptive algorithm using classical criterions (25) and (26). 


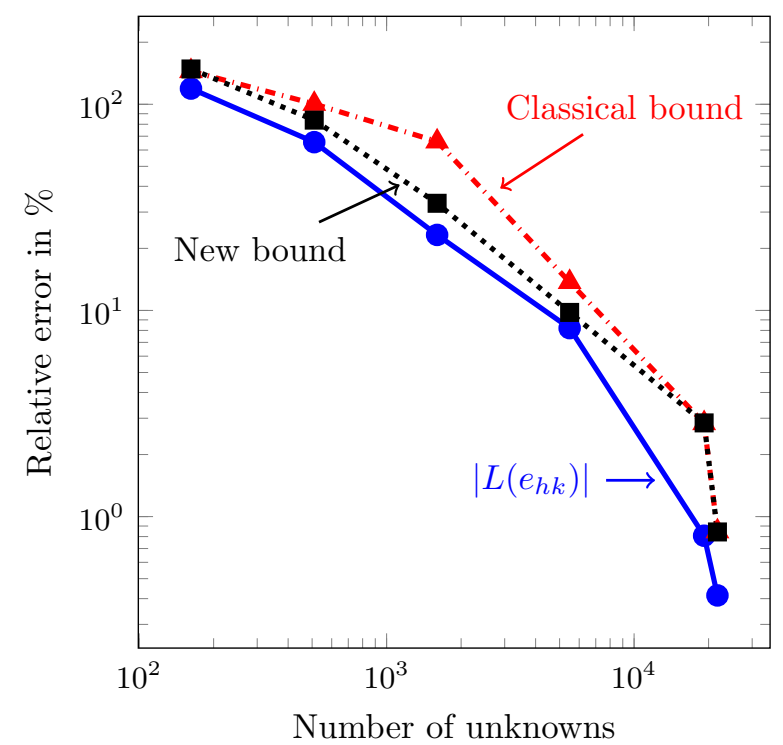

Figure 17: Upper bounds (24) and (27) corresponding to a goal-oriented adaptive algorithm using alternative criterions (28) and (29).
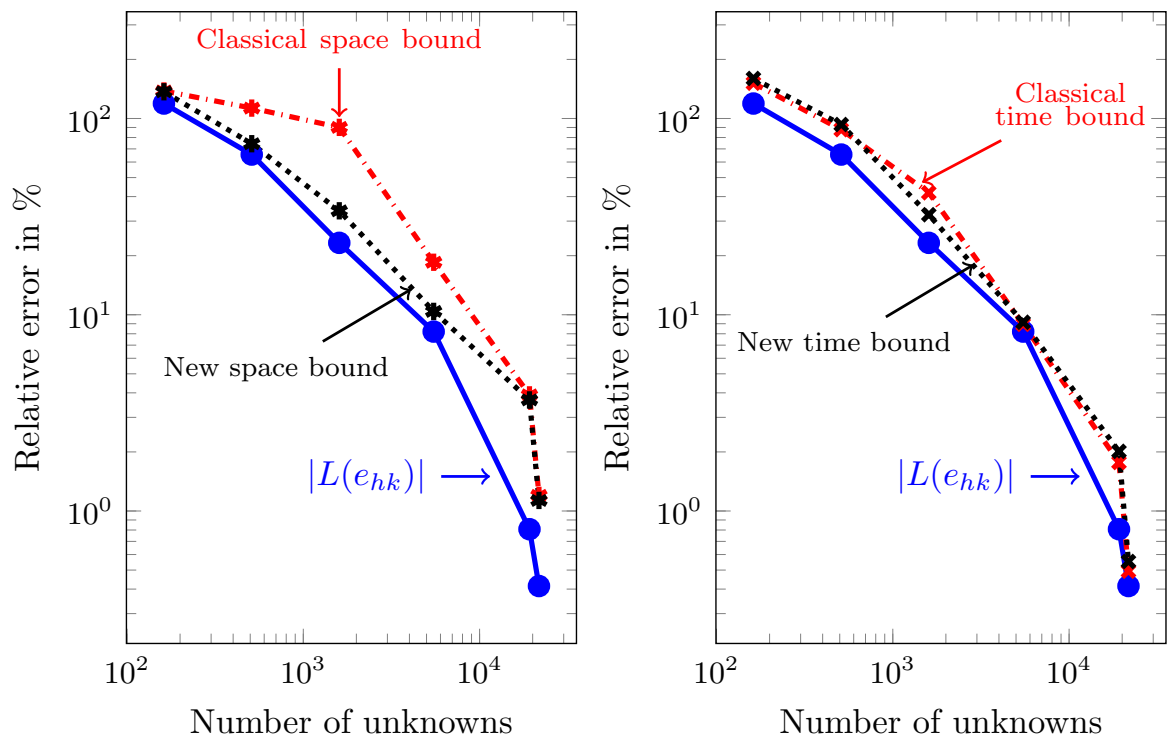

Figure 18: Spatial contribution (left) and temporal contribution (right) of the upper bounds (24) and (27) corresponding to a goal-oriented adaptive algorithm using alternative criterions (28) and (29). 


\section{Conclusions}

We propose an error representation that uses (unconventional) pseudodual problems for goal-oriented adaptivity in time-domain problems. We build a pseudo-dual problem by modifying the standard (classical) dual problem in a way that we obtain another wave propagation problem with better stability properties. We apply this new error representation to the one-dimensional wave equation. We compare the classical upper bounds estimates of the error in the quantity of interest with the new ones, observing that the new bounds are sharper when applied to the $1 \mathrm{D}$ wave equation.

Possible extensions of this work are the study of alternative pseudodual bilinear forms to perform goal-oriented adaptivity and the application of the above ideas to $2 \mathrm{D}$ and $3 \mathrm{D}$ problems. Another possible extension is the reduction of the computational complexity of the existing goal-oriented adaptive strategies based on time-marching schemes by defining pseudo-dual problems that, as the primal problem, evolve forward in time.

\section{Acknowledgments}

The first three authors were partially funded by the Basque Government Consolidated Research Group Grant IT649-13 on "Mathematical Modeling, Simulation, and Industrial Applications (M2SI)" and the Projects of the Spanish Ministry of Economy and Competitiveness with reference MTM2016-76329-R (AEI/FEDER, EU).

All authors have received funding from the European Union's Horizon 2020 research and innovation programme under the Marie Sklodowska-Curie grant agreement No 644602.

David Pardo has also received funding from the Projects of the Spanish Ministry of Economy and Competitiveness with reference MTM2013-40824$\mathrm{P}$ and MTM2016-81697-ERC/AEI, the BCAM "Severo Ochoa" accreditation of excellence SEV-2013-0323 and the Basque Government through the BERC 2014-2017 program.

Victor M. Calo was partially funded by CSIRO Professorial Chair in Computational Geoscience at Curtin University, the National Priorities Research Program grant 7-1482-1-278, the Qatar National Research Fund (a member of The Qatar Foundation) and the Deep Earth Imaging Enterprise Future Science Platforms of the Commonwealth Scientific Industrial Research Organisation, CSIRO, of Australia.

Judit Muñoz-Matute has received founding from the University of the Basque Country (UPV/EHU) grant No. PIF15/346. 


\section{References}

[1] A. Ern, J. L. Guermond, Theory and practice of finite elements, Vol. 159, Springer Science \& Business Media, 2013.

[2] C. Johnson, Numerical solution of partial differential equations by the finite element method, Courier Corporation, 2012.

[3] S. Larsson, V. Thomée, Partial differential equations with numerical methods, Vol. 45, Springer Science \& Business Media, 2008.

[4] G. Strang, G. J. Fix, An analysis of the finite element method, Vol. 212, Prentice-Hall Englewood Cliffs, NJ, 1973.

[5] L. Demkowicz, Computing with hp-adaptive finite elements: Volume 1 One and Two Dimensional Elliptic and Maxwell problems, CRC Press, 2006.

[6] R. Becker, R. Rannacher, A feed-back approach to error control in finite element methods: Basic analysis and examples, Citeseer, 1996.

[7] R. Becker, R. Rannacher, Weighted a posteriori error control in FE methods, IWR, 1996.

[8] J. T. Oden, S. Prudhomme, Goal-oriented error estimation and adaptivity for the finite element method, Computers \& Mathematics with Applications 41 (5) (2001) 735-756.

[9] J. T. Oden, S. Prudhomme, Estimation of modeling error in computational mechanics, Journal of Computational Physics 182 (2) (2002) $496-515$.

[10] S. Prudhomme, J. T. Oden, On goal-oriented error estimation for elliptic problems: application to the control of pointwise errors, Computer Methods in Applied Mechanics and Engineering 176 (1) (1999) 313-331.

[11] S. Prudhomme, J. T. Oden, Computable error estimators and adaptive techniques for fluid flow problems, Error estimation and adaptive discretization methods in computational fluid dynamics 25 (2003) 207268.

[12] J. Alvarez-Aramberri, D. Pardo, H. Barucq, Inversion of magnetotelluric measurements using multigoal oriented hp-adaptivity, Procedia Computer Science 18 (2013) 1564-1573. 
[13] L. Chamoin, P. Ladevèze, A non-intrusive method for the calculation of strict and efficient bounds of calculated outputs of interest in linear viscoelasticity problems, Computer Methods in Applied Mechanics and Engineering 197 (9) (2008) 994-1014.

[14] A. Romkes, J. T. Oden, K. Vemaganti, Multi-scale goal-oriented adaptive modeling of random heterogeneous materials, Mechanics of materials 38 (8) (2006) 859-872.

[15] K. Van der Zee, E. Van Brummelen, I. Akkerman, R. De Borst, Goaloriented error estimation and adaptivity for fluid-structure interaction using exact linearized adjoints, Computer Methods in Applied Mechanics and Engineering 200 (37) (2011) 2738-2757.

[16] C. V. Verhoosel, G. Van Zwieten, B. Van Rietbergen, R. De Borst, Image-based goal-oriented adaptive isogeometric analysis with application to the micro-mechanical modeling of trabecular bone, Computer Methods in Applied Mechanics and Engineering 284 (2015) 138-164.

[17] P. Šolin, L. Demkowicz, Goal-oriented hp-adaptivity for elliptic problems, Computer Methods in Applied Mechanics and Engineering 193 (6) (2004) 449-468.

[18] V. Darrigrand, D. Pardo, I. Muga, Goal-oriented adaptivity using unconventional error representations for the 1D Helmholtz equation, Computers \& Mathematics with Applications 69 (9) (2015) 964-979.

[19] G. Cohen, Higher-order numerical methods for transient wave equations, Springer Science \& Business Media, 2013.

[20] T. J. Hughes, The finite element method: linear static and dynamic finite element analysis, Courier Dover Publications, 2012.

[21] E. Alberdi, J. J. Anza, Solution of the wave-type PDE by numerical damping control multistep methods, Procedia Computer Science 29 (2014) 779-789.

[22] H. Brezis, Functional analysis, Sobolev spaces and partial differential equations, Springer Science \& Business Media, 2010.

[23] J. L. Lions, Optimal control of systems governed by partial differential equations, Vol. 170, Springer Verlag, 1971. 
[24] S. Salsa, Partial differential equations in action: from modelling to theory, Springer Science \& Business Media, 2008.

[25] J. C. Butcher, Numerical methods for ordinary differential equations in the 20th century, Journal of Computational and Applied Mathematics 125 (1) (2000) 1-29.

[26] E. Hairer, S. Nørsett, G. Wanner, Solving ordinary differential equations I. nonstiff problems (1987).

[27] G. Wanner, E. Hairer, Solving ordinary differential equations II, Vol. 1, Springer-Verlag, Berlin, 1991.

[28] H. M. Hilber, T. J. Hughes, R. L. Taylor, Improved numerical dissipation for time integration algorithms in structural dynamics, Earthquake Engineering \& Structural Dynamics 5 (3) (1977) 283-292.

[29] W. Wood, M. Bossak, O. Zienkiewicz, An alpha modification of Newmark's method, International Journal for Numerical Methods in Engineering 15 (10) (1980) 1562-1566.

[30] J. Chung, G. Hulbert, A time integration algorithm for structural dynamics with improved numerical dissipation: the generalized- $\alpha$ method, Journal of applied mechanics 60 (2) (1993) 371-375.

[31] K. E. Jansen, C. H. Whiting, G. M. Hulbert, A generalized- $\alpha$ method for integrating the filtered Navier-Stokes equations with a stabilized finite element method, Computer Methods in Applied Mechanics and Engineering 190 (3) (2000) 305-319.

[32] C. Bernardi, E. Süli, Time and space adaptivity for the second-order wave equation, Mathematical Models and Methods in Applied Sciences 15 (02) (2005) 199-225.

[33] N. Collier, H. Radwan, L. Dalcin, V. M. Calo, Time adaptivity in the diffusive wave approximation to the shallow water equations, Journal of Computational Science 4 (3) (2013) 152-156.

[34] L. Bales, I. Lasiecka, Continuous finite elements in space and time for the nonhomogeneous wave equation, Computers \& Mathematics with Applications 27 (3) (1994) 91-102.

[35] D. A. French, A space-time finite element method for the wave equation, Computer methods in applied mechanics and engineering 107 (1) (1993) $145-157$. 
[36] C. Johnson, Discontinuous Galerkin finite element methods for second order hyperbolic problems, Computer Methods in Applied Mechanics and Engineering 107 (1) (1993) 117-129.

[37] G. M. Hulbert, T. J. Hughes, Space-time finite element methods for second-order hyperbolic equations, Computer Methods in Applied Mechanics and Engineering 84 (3) (1990) 327-348.

[38] M. Morandi Cecchi, R. Nociforo, Discrete finite elements method in space-time domain for parabolic linear problems, Le Matematiche 46 (2) (1993) 655-673.

[39] F. Verdugo, N. Parés, P. Díez, Error assessment in structural transient dynamics, Archives of Computational Methods in Engineering 21 (1) (2014) 59-90.

[40] F. Verdugo, N. Parés, P. Díez, Goal-oriented space-time adaptivity for transient dynamics using a modal description of the adjoint solution, Computational Mechanics 54 (2) (2014) 331-352.

[41] G. Şimşek, X. Wu, K. Van der Zee, E. van Brummelen, Duality-based two-level error estimation for time-dependent PDEs: Application to linear and nonlinear parabolic equations, Computer Methods in Applied Mechanics and Engineering 288 (2015) 83-109.

[42] P. Díez, G. Calderón, Goal-oriented error estimation for transient parabolic problems, Computational Mechanics 39 (5) (2007) 631-646.

[43] N. Parés, P. Díez, A. Huerta, Bounds of functional outputs for parabolic problems. Part I: Exact bounds of the discontinuous Galerkin time discretization, Computer Methods in Applied Mechanics and Engineering 197 (19) (2008) 1641-1660.

[44] M. Schmich, B. Vexler, Adaptivity with dynamic meshes for space-time finite element discretizations of parabolic equations, SIAM Journal on Scientific Computing 30 (1) (2008) 369-393.

[45] W. Bangerth, M. Geiger, R. Rannacher, Adaptive Galerkin finite element methods for the wave equation, Computational Methods in Applied Mathematics 10 (1) (2010) 3-48.

[46] W. Bangerth, R. Rannacher, Finite element approximation of the acoustic wave equation: error control and mesh adaptation, East West Journal of Numerical Mathematics 7 (4) (1999) 263-282. 
[47] W. Bangerth, R. Rannacher, Adaptive finite element methods for differential equations, Birkhäuser, 2013.

[48] D. Pardo, L. Demkowicz, C. Torres-Verdín, L. Tabarovsky, A goaloriented hp-adaptive finite element method with electromagnetic applications. Part I: Electrostatics, International Journal for Numerical Methods in Engineering 65 (8) (2006) 1269-1309.

[49] D. Pardo, L. Demkowicz, C. Torres-Verdin, M. Paszynski, A selfadaptive goal-oriented hp-finite element method with electromagnetic applications. Part II: Electrodynamics, Computer methods in applied mechanics and engineering 196 (37) (2007) 3585-3597. 